\title{
Liame
}

Histoire et histoire de l'art des époques moderne et contemporaine de l'Europe méditerranéenne et de ses périphéries

25 | 2012

Les réseaux négociants dans la France méridionale (XVIII ${ }^{\mathrm{e}} \mathrm{XIX} \mathrm{X}^{\mathrm{e}}$ siècles)

\section{Les négociants de la France méridionale à Bordeaux entre la fin de l'Ancien Régime et la Restauration}

\section{Philippe Gardey}

\section{Q OpenEdition}

\section{Journals}

Édition électronique

URL : http://journals.openedition.org/liame/261

DOI : 10.4000/liame.261

ISSN : 2264-623X

Éditeur

CRISES - Centre de Recherches Interdisciplinaires en Sciences Humaines et Sociales de Montpellier

Référence électronique

Philippe Gardey, « Les négociants de la France méridionale à Bordeaux entre la fin de l'Ancien Régime et la Restauration », Liame [En ligne], 25 | 2012, mis en ligne le 13 décembre 2012, consulté le 13 novembre 2019. URL : http://journals.openedition.org/liame/261 ; DOI : 10.4000/liame.261

Ce document a été généré automatiquement le 13 novembre 2019.

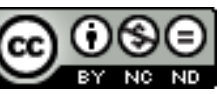

Les contenus de Liame sont mis à disposition selon les termes de la Licence Creative Commons Attribution - Pas d'Utilisation Commerciale - Pas de Modification 4.0 International. 


\title{
Les négociants de la France méridionale à Bordeaux entre la fin de l'Ancien Régime et la Restauration
}

\author{
Philippe Gardey
}

1 De la fin de l'Ancien Régime au début du XIX ${ }^{e}$ siècle, la richesse de Bordeaux reposa en grande partie sur l'activité d'hommes venus de régions fort éloignées du grand port aquitain. On pense aux commissionnaires hollandais, germaniques, anglo-irlandais ou encore aux juifs portugais qui animèrent la croissance $d u$ siècle d'or. Si nombre de très grands négociants furent, en effet, des hommes originaires de l'Europe du Nord ou de la diaspora israélite, il ne faut pas oublier que l'essentiel des migrants, y compris dans les milieux du commerce, venaient alors des profondeurs du royaume. Parmi ces derniers, ceux de la moitié méridionale de la France dominaient largement. Issus d'un vaste arrière-pays qui s'étendait jusqu'à la Méditerranée, ils contribuaient à la constitution des réseaux commerciaux qui alimentaient Bordeaux en produits manufacturés comme en productions de la terre. Ils étaient les relais indispensables entre ce riche interland et la communauté cosmopolite des commissionnaires qui, depuis Bordeaux, approvisionnaient les îles et l'Europe du Nord.

2 Aborder une telle question autrement que de manière impressionniste, oblige à mesurer l'ampleur du flux migratoire des négociants méridionaux vers Bordeaux, puis à dresser une carte, la plus précise possible, de leurs origines pour mettre en évidence les liens entre ces flux migratoires et les logiques commerciales bordelaises. Cette approche doit être complétée par l'établissement d'une sorte de profil type du négociant méridional à Bordeaux. Était-il un héritier? Possédait-il des moyens financiers importants en arrivant? Quelles étaient d'ailleurs les modalités les plus répandues de son installation dans le grand port aquitain?

3 Nous nous proposons d'approfondir ici ces thèmes au moyen de sources sérielles bordelaises que sont les actes notariés. Enrichis par plusieurs sources fiscales, les riches 
dossiers de la Commission militaire de l'an II et les listes de commerçants des almanachs bordelais, ils nous permettent de tenter une approche prosopographique de la question. $\mathrm{Au}$-delà de mises en perspectives statistiques toujours nécessaires mais un peu arides, la reconstitution de nombre de trajectoires individuelles et familiales fournit un contrepoint utile et donne un peu de chair à cette question des réseaux commerciaux entre la France méridionale et Bordeaux. Au travers de deux cas très significatifs, nous pourrons alors tenter, une approche des logiques et des stratégies qui présidaient à la constitution de réseaux négociants dans lesquelles préoccupations familiales et commerciales s'entremêlaient presque toujours.

\section{L'ampleur du flux migratoire négociant vers Bordeaux et son rythme}

Depuis les travaux de Jean-Pierre Poussou, le rôle des flux migratoires dans la croissance bordelaise de la seconde moitié du XVIII ${ }^{\mathrm{e}}$ siècle n'est plus à démontrer ${ }^{1}$. Si dans les années 1750 près de $60 \%$ des négociants qui se mariaient à Bordeaux y étaient nés ${ }^{2}$, ils n'étaient plus que $31,7 \%$ entre 1784 et 1800 . Cette accélération, comparable à la croissance de l'activité économique qu'elle contribuait d'ailleurs à nourrir, ne fut d'ailleurs que partiellement remise en cause par la crise révolutionnaire et impériale. Ainsi, entre 1808 et 1825 , les Bordelais ne représentaient toujours que $40,7 \%$ des négociants qui se mariaient. Près des deux tiers des hommes des milieux négociants étaient donc des étrangers à la ville et comme assez peu provenaient du reste de la Gironde (6 à $7 \%$ ), le bassin de recrutement des milieux du commerce formait une aire extrêmement vaste dans laquelle la France méridionale pesait d'un poids très lourd ${ }^{3}$.

5 Si on la considère dans sa plus vaste extension, c'est-à-dire au Sud d'une ligne qui irait de la Rochelle à Lyon, et si on prend en compte les mariages des années 1780-1820, elle fournissait alors $39 \%$ du total des négociants au mariage et $61 \%$ des non Bordelais ${ }^{4}$. À cette moisson de 201 contrats de méridionaux venant se marier à Bordeaux, il faut ajouter 62 contrats concernant des méridionales venant à Bordeaux pour épouser des négociants. Les femmes représentaient traditionnellement une immigration beaucoup plus faible et circonscrite que les hommes, ce qui fait qu'à Bordeaux 65,6 \% des épouses de négociants sont alors des Bordelaises. Ainsi, ces 62 méridionales pèsent lourd dans nos chiffres avec $50,4 \%$ des immigrantes qui viennent épouser un négociant à Bordeaux.

6 Le rythme de cette immigration est assez intéressant. À l'échelle globale nous avons déjà fait le constat classique d'un apogée de l'attraction bordelaise à la fin du XviII siècle (1784-1800) suivi par un resserrement modéré des horizons migratoires au début du siècle suivant (1808-1825)5. L'amenuisement est très clair pour les marchands. Les négociants résistent mieux aux soubresauts de l'époque, ce qui prouve que sous l'Empire et durant la Restauration on pouvait encore avoir l'espoir de s'enrichir dans le grand commerce. Plus que d'un amenuisement, il faudrait parler, à leur égard, d'un resserrement sur les zones les plus proches, c'est-à-dire, justement cette France méridionale que nous étudions ici. Du Haut-Pays au Languedoc, elle correspond aux amarres terriennes de Bordeaux, un interland qui fournit les denrées alimentaires et les produits manufacturés approvisionnant les colonies ainsi que les hommes qui animent ce commerce. 
7 Une étude détaillée par coupes nous permet d'approfondir l'analyse à l'échelle de cette seule France méridionale.

Figure 1 : migrations de méridionaux dans le négoce bordelais d'après les contrats de mariages

\begin{tabular}{|l|l|l|l|l|l|}
\hline & $1784-1788$ & an III-an VIII & $1808-1812$ & $1821-1825$ & Total \\
\hline Hommes & 41 & 77 & 38 & 45 & 201 \\
\hline Femmes & 4 & 33 & 9 & 16 & 62 \\
\hline Total & 45 & 110 & 47 & 61 & 263 \\
\hline Moyenne annuelle & 9 & 18,3 & 9,4 & 12,2 & 12,6 \\
\hline
\end{tabular}

8 Le flux migratoire en provenance de l'arrière-pays bordelais semble autonome par rapport à la conjoncture économique. On ne constate pas de fléchissement durant la période révolutionnaire, ni même, entre 1808 et 1812, au pire de la crise impériale. Ce qui étonne, en revanche, c'est le doublement de la moyenne durant le Directoire. La raison en est le rattrapage en l'an III et en l'an IV des mariages qui n'avaient pas pu se conclure durant la Terreur. La moyenne des années qui vont de l'an v à l'an viII retombe, en effet à 10,2, ce qui est tout à fait comparable aux moyennes des autres périodes. C'est donc finalement la stabilité qui l'emporte. Chaque année, de la fin de l'Ancien Régime, à la Restauration, Bordeaux draine une dizaine de méridionaux issus des milieux du négoce. Sur 50 ans cela représente, sans doute, plus de 500 hommes et femmes des milieux du commerce attirés par le dynamisme du grand port atlantique.

\section{L'axe garonnais, principal pourvoyeur de négociants méridionaux à Bordeaux}

9 Tout réseau marchand lie ensemble un certain nombre d'individus appartenant ou non à la même firme. Ces agents, ces fournisseurs, ces commissionnaires, situés en plusieurs points d'un circuit, servent de relais, contrôlent l'approvisionnement, facilitent les débouchés et rendent profitable le bouclage d'opérations parfois complexes. Pour tenir les deux bouts de la corde d'un marché, agir avec confiance ou écarter un concurrent, la famille offre la solution naturellement la plus recherchée. Ainsi la migration des négociants méridionaux à Bordeaux répond toujours à un besoin, permet une nouvelle spéculation, permet l'accès, par un mariage, à un autre réseau de solidarités marchandes. Grâce au dépouillement des notaires bordelais, nous connaissons nominativement près de la moitié de ces méridionaux venus tenter leur chance à Bordeaux. Pour 182 hommes et $60 \mathrm{femmes}$, nous possédons les origines géographiques précises. Nous pouvons ainsi dresser une carte détaillée des provenances sur la longue durée et constater combien elles sont étroitement liées aux activités commerciales. 
Figure 2 : négociants et épouses de négociants bordelais originaires de la France méridionale (1784-1825).

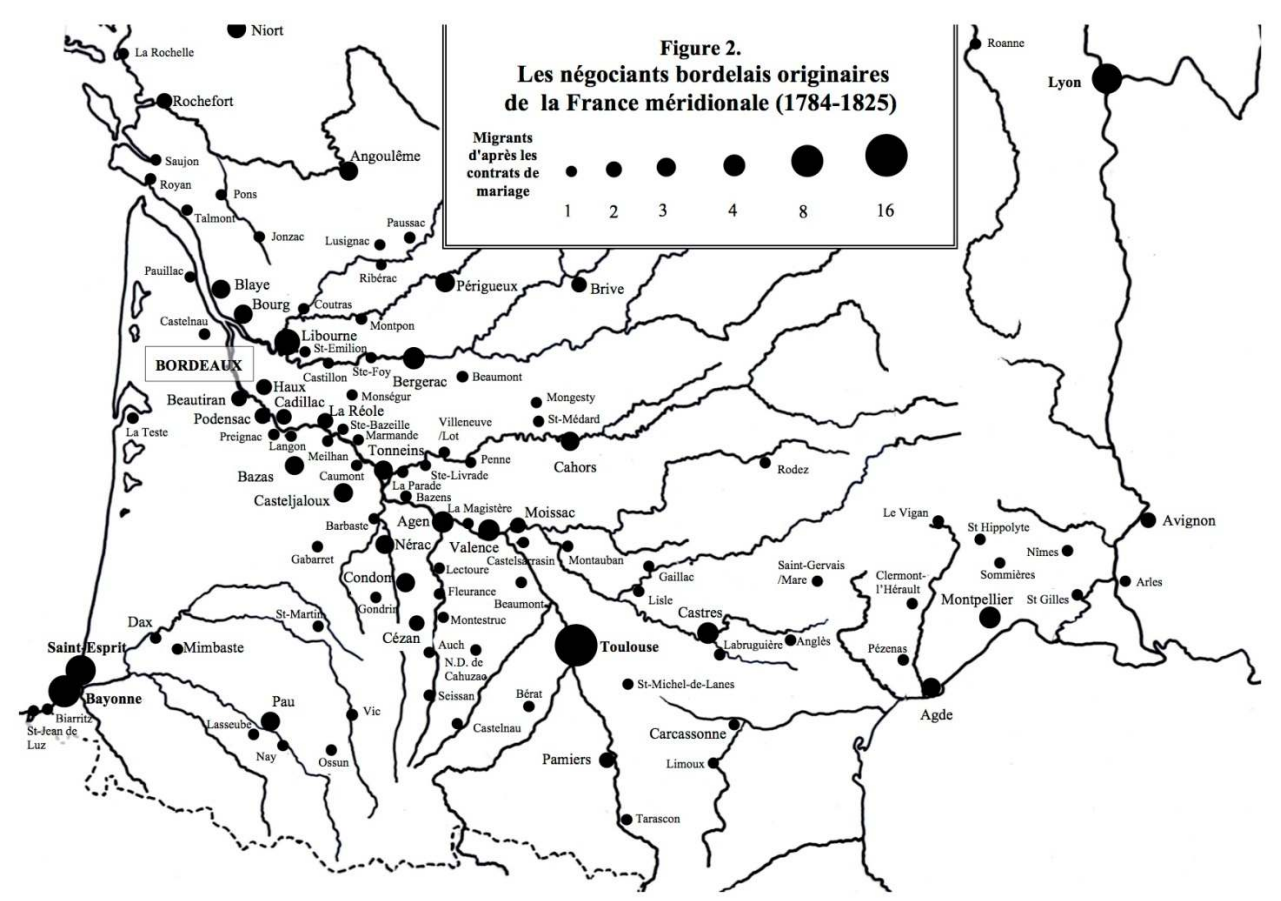

10 Considérons un vaste espace compris entre la côte atlantique de la Rochelle à Bayonne et la vallée du Rhône de Lyon à Avignon. Le premier constat est que pour Bordeaux, la France méridionale entre le Rhône et l'Italie n'existe tout simplement pas. C'est évidemment l'aire d'influence exclusive de Marseille ou de Gênes. La véritable zone de recrutement des négociants à la forme d'un vaste triangle dont les sommets seraient la Rochelle, Bayonne et Avignon. Cet espace, largement ouvert sur l'Atlantique s'étire en se réduisant progressivement entre le piémont pyrénéen et les contreforts du Massif Central. L'organisation n'est pas celle de cercles concentriques mais plutôt d'un axe : celui de la Garonne prolongé par le canal du Midi et les villes du Bas-Languedoc. Au bassin hydrographique garonnais s'ajoutent deux réseaux secondaires; au Nord, celui de la Dordogne, au Sud-Ouest, celui de l'Adour. Les provenances détaillées par localités mettent en évidence deux caractéristiques fondamentales: l'immigration négociante est très majoritairement urbaine et elle se concentre sur les axes fluviaux navigables. Ces derniers constituent alors les grandes voies de transports et les villes qui s'y sont développées sont autant de marchés et de petits ports en relation commerciale étroite avec Bordeaux. Si, pour un emporium colonial de réexportation en plein essor, cet arrière-pays pèse de moins en moins lourd, pour ces localités, c'est un moyen de s'ouvrir au commerce atlantique en orientant les productions locales vers des produits d'exportation'

11 Ces caractéristiques expliquent pour commencer, la place modeste de l'estuaire de la Gironde, en aval du port. Le rôle de la « Rivière de Bordeaux » est tel dans le commerce du port de la lune que l'on pourrait s'attendre à voir des liens nombreux entre ses rives et les hommes qui animent ce commerce. Or il n'en est rien, car il s'agit de régions très rurales peu tournées vers les activités maritimes. Personne n'est originaire du Médoc, hormis Castelnau et Pauillac. La rive droite de la Dordogne, puis de la Gironde à partir du bec d'Ambès, ne fournit pratiquement rien en dehors des deux petites cités commerçantes de 
Saint-André et Bourg et du port de Blaye qui envoient à Bordeaux quelques négociants. De Charente-Maritime proviennent seulement 2,2 \% des négociants. Un seul Rochelais est attiré par Bordeaux en 21 années! Ici, les relations d'affaires ne génèrent pas de flux migratoires. Ce sont finalement les petits ports actifs de Saintonge, de Talmont à Royan qui fournissent à Bordeaux, les rares négociants liés au monde maritime. Il s'agit d'une petite communauté de huguenots qui produit un type de négociant finalement assez rare : le capitaine de navire audacieux qui se lance dans la commission et l'armement ${ }^{7}$.

12 Au Nord, le bassin de la Dordogne envoie à Bordeaux davantage de négociants. Grande cité du vin, c'est Libourne qui fournit les plus gros effectifs. Au-delà, l'immigration n'est sensible que dans quelques villes qui alimentaient le marché hollandais en vins blancs: Castillon, Sainte-Foy et surtout Bergerac. Les provenances se limitent ensuite pour l'essentiel à Coutras et Ribérac sur la Dronne, Périgueux sur l'Isle et Brive sur la Corrèze. Sainte-Foy comme Périgueux produisaient des étoffes de laine et pour les maitres de forge du Périgord, qui fabriquaient, en particulier, des chaudières en fonte destinées aux sucreries antillaises, Bordeaux constituait l'unique débouché.

13 C'est sur la Garonne proprement dite que le recrutement est particulièrement dense jusqu'à la confluence avec le Tarn. Il s'agit de la moyenne Garonne qui s'est spécialisée dans la culture de blés durs destinés à la fabrication de farines connues sous le nom de minots. Elles étaient réclamées par les Îles, car elles supportaient bien le voyage. La plupart des minoteries fabriquaient aussi les biscuits destinés à nourrir les équipages et les esclaves lors de la traversée de l'Atlantique. Bordeaux avait échoué à plusieurs reprises à imposer une prohibition des biscuits du Haut-Pays. La croissance des exportations de ses farines a correspondu à l'essor de l'armement colonial bordelais car, à la veille de la Révolution, ce sont plus de 200.000 barils de 175 livres-poids ${ }^{8}$ chacun qui remontaient la Garonne avant d'être embarqués, contre à peine 100.000 au début des années 1770. C'est donc tout naturellement que les centres de minoterie d'Agen, Valence, Moissac et jusqu'à Montauban alimentent Bordeaux en négociants. Mais Tonneins est en bonne place qui, outre les minots, fournissait des cordages de chanvre et possédait, depuis 1726, une des neuf manufactures royales de tabac du royaume. Quant à Agen, il constituait un petit centre industriel réputé pour sa tannerie qui utilisait l'indigo et les cuirs de Saint-Domingue, quatre manufactures d'impression d'indiennes et une manufacture royale de toiles de chanvre qui alimentait en voiles les vaisseaux du roi. Si la densité est aussi forte, c'est que Bazas, La Réole, Marmande, Casteljaloux, Agen et Valence sont aussi de petits centres de draperie. La laine des moutons du pays produisait des serges, des étamines et des couvertures grossières qui trouvaient un débouché à Bordeaux. Cet éveil industriel était cependant fragile et les régions de la moyenne Garonne entrèrent en crise dès le règne de Louis XVI. La Révolution ne fit qu'aggraver les choses en fermant les débouchés extérieurs. Les flux migratoires devaient cependant se maintenir car, en période de difficultés conjoncturelles, Bordeaux restait finalement le pôle le plus attractif de la région pour de jeunes gens entreprenants.

14 Le recrutement négociant se poursuit sur la partie navigable du Tarn jusqu'à la cité de Gaillac. C'était alors un entrepôt considérable par où transitaient les vins de la région, les farines-minots de l'Albigeois et les draps de Lodève. Les nombreuses petites cités du Lot qui alimentent Bordeaux en vins rouges, eaux-de-vie, prunes et minots donnent aussi des négociants jusqu'à Cahors, ville au-delà de laquelle la rivière n'est plus navigable. Le Lot était bien la rivière des vins comme la Garonne était celle des blés. Certains négociants 
protestants de la région ont leurs propres correspondants en Europe du Nord pour écouler les vins, mais le débouché bordelais reste essentiel' ${ }^{9}$.

Dans le Haut-Armagnac, les affluents de la rive gauche de la Garonne, moins importants économiquement, alimentent cependant un courant migratoire non négligeable : Nérac, centre de minoterie, de draperie et de tannerie ainsi que Condom sur la Baïse (eaux-devie) mais aussi Lectoure, Fleurance, Montestruc, Auch et Seissan sur le Gers.

À l'Est et au Sud de Toulouse, les négociants proviennent encore du centre drapier de Castres et de ses environs sur l'Agout, affluent du Tarn, et de Pamiers ou Tarascon sur l'Ariège. Toulouse enfin constituait la véritable porte du Languedoc. C'est là que s'opérait la rupture de charge du transport. Les marchandises provenant d'Agde par le canal des Deux-Mers y étaient débarquées avant d'être réparties sur les embarcations venant de Montauban, Moissac et Agen. Ce n'est donc pas un hasard si cette ville de près de 50000 habitants fournit, après Bayonne, les plus gros effectifs de négociants venus s'installer et se marier à Bordeaux entre 1784 et 1825. Parfois, l'alliance ne nécessite pas la migration définitive du négociant. Quand Jean-Baptiste Delmon, épouse Henriette Raba, la fille d'Antoine-Henriques Raba, un des cinq frères Raba originaires du Portugal, il exerce toujours à Toulouse et nous ne trouvons pas trace d'une société à son nom à Bordeaux ${ }^{10}$. Les élites du négoce toulousain n'hésitent pas à envoyer des enfants à Bordeaux. C'est le cas des Pelissier. Le père de Jean-François-Zacharie Pelissier était ainsi consul de la Bourse de Toulouse avant la Révolution ${ }^{11}$. Un cas classique de migration à Bordeaux est représenté par les Domecq. Le père, Barthélémy, né en 1731, était négociant à Toulouse, rue de la Borle, dans la paroisse commerçante de la Daurade. Il y est encore à la naissance de son fils Clément en $1767^{12}$. C'est seulement à la veille de la Révolution qu'il s'installe à Bordeaux, rue du Chai des farines. Le $1^{\text {er }}$ mai 1788, il associe son fils à ses affaires par un acte sous-seing privé, sous la raison Domecq père \& fils ${ }^{13}$. En 1789, c'est un commissionnaire qui vend aux Antilles tous les produits de l'arrière-pays garonnais et redistribue ses marchandises coloniales vers les ports du Nord comme Amsterdam et Rotterdam ${ }^{14}$

17 Le courant migratoire vers Bordeaux se prolonge ensuite le long du canal des Deux-Mers par Carcassonne et Agde. C'est par la voie maritime qu'on rejoint alors Marseille ou la vallée du Rhône avant le prolongement du canal en 1805. Il emprunte aussi la voie terrestre qui, depuis Gaillac et Albi, gagne Lodève et Nîmes. Ces voies convergent toutes vers Beaucaire, où se tiennent des foires très réputées. L'importance économique de la région explique que, très loin de Bordeaux, le recrutement y soit remarquable. Il concerne non seulement les grands centres comme Agde, Montpellier, Nîmes, Arles, Avignon, mais aussi les villes de foires régionales comme Pézenas et Saint-Gilles, ou les bourgs animés comme Le Vigan (bas de soie), Sommières (cuirs et étoffes de laine), StHippolyte-du-Fort (bas de soie), Clermont-l'Hérault (draps pour l'armée).

18 Au Sud-Ouest, enfin, le bassin de l'Adour représente la dernière zone de recrutement du négoce bordelais. En vérité, il compte surtout par la place exceptionnelle de Bayonne qui devance Toulouse avec 18 représentants du négoce qui viennent s'installer et se marier à Bordeaux entre 1784 et 1825 . Le trafic colonial de Bayonne, qui rivalisait encore avec celui de Bordeaux au début du règne de Louis XV s'est laissé distancer pour devenir 14 fois inférieur dans les années $1780^{15}$. La ville ne constitue plus alors un pôle de croissance commerciale suffisant pour retenir ses négociants. Dans l'arrière-pays, il faut aller jusqu'au cœur du Béarn autour de Pau et de Nay pour trouver un petit pôle de 
recrutement. Il met le port aquitain en relation avec une région textile spécialisée dans les toiles et les mouchoirs de lin.

\section{Immigration négociante et places financières de la France méridionale}

Cette première approche peut être utilement complétée par une comparaison entre les origines géographiques des négociants et les lettres de change protestées à Bordeaux durant la même période. Ici encore, les notaires nous sont d'un grand secours grâce à un acte que l'on trouve très souvent dans les minutes : le protêt. Il concerne les lettres de change et il est dressé faute d'acceptation ou faute de paiement à l'échéance. L'intérêt pour l'historien est que la lettre de change concernée est intégralement recopiée au début de l'acte. Nous avons ainsi accès à des milliers d'effets de commerce dont les originaux ont disparu depuis longtemps. Sur 25 années ; on repère ainsi 795 lettres de change tirées hors de Bordeaux ${ }^{16}$. Sur ce total, 212 concernent des localités de la France méridionale soit un peu plus du quart. L'essentiel des opérations financières concerne, en effet, les îles et les grandes places de l'Europe du Nord, avant la montée en puissance de Paris à partir de la Révolution ${ }^{17}$.

Figure 3 : lettres de Change concernant la France méridionale protestées à Bordeaux entre 1784 et 1825.

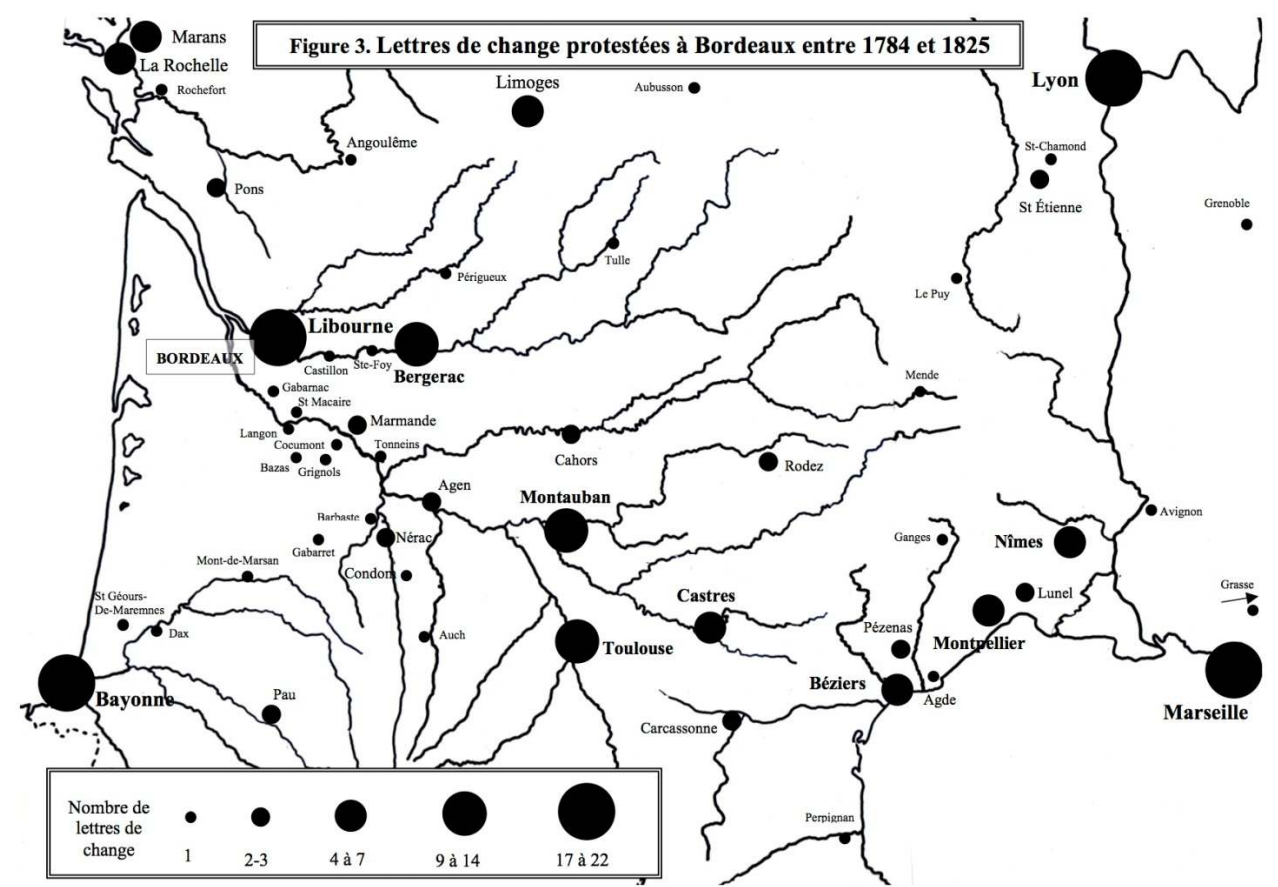

C'est une géographie assez semblable à celle des flux migratoires que nous voyons apparaître. L'importance des relations avec les négociants de Libourne et de Bergerac ressort avec davantage de force encore. Libourne, au débouché de la Dordogne, domine le commerce du Haut-Pays. Les Libournais ont leur propre réseau: une trentaine de négociants liés à des courtiers qui les mettent en contact avec les propriétaires de Pomerol, de Saint-Émilion et de Castillon. C'est un monde relativement indépendant de Bordeaux. Les annuaires bordelais donnent d'ailleurs des listes séparées des négociants et 
courtiers libournais. Malgré tout, les marchés étrangers de Libourne s'effondrent après la guerre d'Amérique et désormais, pour un Libournais, l'horizon international passe par Bordeaux ${ }^{18}$.

21 Le bassin de la Garonne représente bien l'axe fondamental de l'arrière-pays bordelais. La place de Montauban envoie assez peu de négociants à Bordeaux, mais concentre les transactions financières de la moyenne Garonne. À la fin de l'Ancien Régime, elle est devenue une véritable cité industrielle et ouvrière de 25.000 habitants. On y trouve près de 60 maisons de négoce. La minoterie (une vingtaine d'entreprises) y a supplanté les autres centres du Sud-Ouest et une quinzaine de fabricants-drapiers emploient des milliers de paysans dans les campagnes proches. L'armateur bordelais peut directement passer commande aux minotiers locaux ou passer par l'intermédiaire d'un commissionnaire. Nombre de minotiers empruntent à l'armement et deviennent ainsi dépendants du négoce bordelais ${ }^{19}$.

Le rôle de Toulouse est, en revanche tout à fait proportionnel à son poids dans les migrations. Comme nous l'avons vu, la ville constituait un relais indispensable entre la Bas-Languedoc industriel et le port colonial de Bordeaux qui exportait une partie de sa production. Les maisons de Bordeaux avaient intéret à s'associer à celles de Toulouse qui leur servaient de relais commercial et financier. Nombre de sociétés toulousaines sont en relation avec Bordeaux comme Lourde, Martignac \& Cie, Delmon père \& fils, L'Hospital, Martignac \& Pelissier.

C'est tout le Bas-Languedoc qui s'anime ensuite sous nos yeux. Le débouché du canal du Midi avec Béziers et Agde, la vallée de l'Hérault de Pézenas à Ganges. Mais aussi Montpellier, Lunel et Nîmes. Cette dernière ville ressort davantage que dans les courants migratoires car le port de Nîmes, c'est Beaucaire. C'est là que chaque année, en juillet, les foires attirent près de 100.000 personnes en dix jours et génèrent un chiffre d'affaires de près de 50 millions de livres à l'extrême fin $\mathrm{du}$ xvIII ${ }^{\mathrm{e}}$ siècle. Sucres raffinés et cafés provenant de Bordeaux y sont échangés contre les draperies languedociennes et les produits provençaux. Dans les dernières années de l'Ancien Régime, Bordeaux avec $600.000 \mathrm{Et}$, y vend plus que Marseille ${ }^{20}$. Les réseaux relationnels entre Bordeaux et Montpellier sont intenses et durables. Les lettres de change du Directoire font ainsi apparaître les liens entre la société Veuve Raspay de Montpellier et la maison Raspay \& Droz établie à Bordeaux. En 1813, encore, les commissionnaires allemands des Chartrons Clossmann \& Cie font appel à Raspay \& Cie pour leur fournir de la crème de tartre, du vert-de-gris ou de l'esprit-de-sel du Bas-Languedoc industriel, mais aussi des amandes de la vallée de l'Hérault. La maison bordelaise traite directement en se passant de l'intermédiaire toulousain sans doute trop coûteux en période de crise. De son côté, le négociant de Montpellier auquel elle s'adresse, fait tout pour pérenniser des échanges qui lui assurent des débouchés bienvenus. «Faites souvent usage de nos services » conclut-til dans une de ses missives «nous vous en réitérons l'offre de bon cœur, et vous saluons bien affectueusement $»^{21}$.

Marseille enfin, avec 17 lettres apparaît comme une des principales places en rapport avec Bordeaux alors que pas un des 655 contrats de mariage sur les 21 années que nous avons dépouillées, entre 1784 et 1825 , ne mentionne un négociant originaire de la cité phocéenne. C'est bien au-delà de Marseille que Bordeaux attire quelques négociants comme l'armateur Pierre Susini de Livourne ${ }^{22}$, Gérome Chiapella né près de Gênes $^{23}$ ou encore Marie Roques Parmeygiani de Reggio ${ }^{24}$. Mais, la faiblesse des liens migratoires ne signifie pas ici l'absence de relations commerciales. Les négociants 
marseillais investissent dans l'armement bordelais comme ceux de Nantes ou de SaintMalo. On les repère dans les actes d'abandon de navire. Cela ne donne, bien sur, qu'une faible idée des investissements du négoce français dans l'armement bordelais car nombre d'assureurs agissent, par procuration, pour le compte de «marques» rarement identifiables.

À Lyon, l'importance de la place financière dépasse de loin celle des flux migratoires. Les protêts nous livrent les noms de nombreuses maisons comme Jacques Lassère (banque), Fitler \& Darnal (banque), Reynaud, Genissieux \& Massot frères (marchands drapiers), Flahault \& Olive, Louis \& Bignan, Jh Mallié, Pliney frères ${ }^{25}$. Mais, à la différence de Marseille, Lyon envoie quelques négociants à Bordeaux comme Lazare Chaulet (fils d'un agent de change) et Melchior Lacorée avant la Révolution, ou André Gachet et Antoine Seriziat (famille de fabricant de soieries) durant le Directoire. Cependant, ce sont surtout des Lyonnaises qui viennent s'installer à Bordeaux : quelques filles de négociants comme Jeanne Martin ou Gabrielle Grenier, mais surtout des marchandes, comme les sœurs Mamy. Filles d'un marchand de Lyon, deux s'installent à Bordeaux comme marchandes de mode et la troisième comme marchande de toiles. Durant la Révolution, elles possèdent des fonds de commerce de 30.000 à $50.000 \mathrm{ft}$, niveau considérable dans leurs spécialités, qui leur permettent d'épouser des négociants.

La Rochelle et Limoges enfin présentent, elles aussi, le cas de villes intégrées à l'aire du commerce bordelais mais dont les négociants éprouvent peu ou pas du tout le besoin de venir s'installer à Bordeaux. Pour Limoges, ce sont des marchands forains qui viennent aux foires de Bordeaux et y redistribuent la production des grandes régions textiles du Nord de la France. Quant au port de La Rochelle, il offre des possibilités de réussite locale très importante dans le commerce colonial et la traite négrière avant la Révolution. Les maisons bordelaises sont, en revanche, en contact avec les maisons rochelaises pour le commerce des eaux-de-vie de Cognac. C'est d'ailleurs pour la même raison qu'on voit apparaitre le port de Marans, port du sel, mais qui concurrence La Rochelle pour l'exportation des eaux-de-vie de l'Aunis ${ }^{26}$.

Avec Bayonne, la coïncidence des deux cartes est, en revanche, frappante. Mais ici, bien plus que des relations commerciales relativement modestes, ce sont les solidarités religieuses de la diaspora israélite qui jouent pleinement. Toute alliance avec les autres groupes du négoce est exclue car les unions mixtes ne sont pas admises par les rabbins. Le sang neuf ne peut être apporté que par des conjoints que l'on va chercher hors de Bordeaux. Nombre de juifs séfarades de «la nation portugaise» s'étaient installés à Bayonne, dans le faubourg Saint-Esprit, avant de s'établir à Bordeaux. On trouve ainsi de véritables nébuleuses familiales de négociants et banquiers partagées entre les deux villes comme les Fonsèque, Gommes, Julian, Léon, Oxeda, Pereyra-Soares, Rodrigues ou encore Roget. Elles alimentent Bordeaux en piastres provenant de l'empire espagnol.

\section{Le rôle fondamental des filières d'immigration calvinistes}

Dans cette France méridionale, plus encore que la diaspora israélite, la communauté calviniste joue un rôle particulièrement important. La force des communautés religieuses, en particulier dans le monde du commerce est particulièrement frappante. Entraide, cohésion interne, phénomène de diaspora favorable aux relations commerciales avec les 
régions lointaines : tout concourt à faire de ces groupes des acteurs majeurs de l'activité économique.

Les nombreux négociants dont nous avons pu suivre les destinées nous permettent d'identifier plusieurs courants migratoires qui renouvellent en permanence la communauté protestante bordelaise et permettent de constituer autant de réseaux commerciaux.

Le plus proche vient de Saintonge. Charles-Paul Boudin, marchand de graisseries qui se lance dans l'armement avant la Révolution, était ainsi né à Mortagne. Jean-Elie Gautier qui fait partie des plus gros négociants de la place avant 1789 était de Marennes. La famille Guestier prend ses racines entre Talmont et Meschers. La mère de Daniel Guestier était la sœur de Jacques Conte. Ce dernier, qui allait devenir le plus grand armateur de corsaires de la Révolution, était de Chaillevette et son père, capitaine de navires, de Meschers. Daniel Lys, enfin, était le fils de Jean Lys, un huguenot originaire de Jonzac. Avec son frère, Daniel, il était venu s'installer à Bordeaux comme marchand de toiles avant d'étendre ses activités au négoce et à l'armement.

1 Le second courant suit la vallée de la Dordogne. On y trouve des hommes comme Pierre Texier qui allait marier sa fille à l'Américain Jona Jones. Les Burête, les Couderc et les Boucherie de Bergerac représentent un cas particulièrement intéressant. Facteurs régionaux, professionnels et confessionnels cumulent ici leurs effets. La famille Burète comptait des jurats nobles de Bergerac, cité où elle était connue depuis longtemps. Né dans cette ville en 1745, Jacques Burète était le fils aîné d'un négociant protestant de la cité. Sa mère Elisabeth Boucherie était aussi issue d'une famille du négoce protestant de Bergerac installée à Bordeaux depuis le règne de Louis $\mathrm{XIV}^{27}$. Son oncle, qui se nommait aussi Jacques, est sans doute le premier des Burète à s'être installé à Bordeaux au XVIII siècle. En 1784, Jacques Burète épousa Jeanne-Elisabeth Couderc, la fille du négociant Isaac Couderc jeune ${ }^{28}$. Or les Couderc étaient aussi des protestants de Bergerac établis à Bordeaux à la fin du XVII ${ }^{\mathrm{e}}$ siècle. Burète se liait ainsi à ses deux beaux-frères associés dans la maison de négoce et d'armement Couderc frères. L'aîné, François, avait d'ailleurs épousé une Bergeracoise un mois avant ${ }^{29}$. La mère de la future se nommait Elisabeth Boucherie, tout comme la mère de Burète. Ce dernier épousait sans doute une cousine. La tante de la future, Marthe Boucherie, était aussi l'épouse de l'armateur protestant Jacques Delorthe. Ce n'est donc pas un hasard si, avec sa femme et ses six enfants, il est à la signature du contrat. Les solidarités géographiques et confessionnelles sont donc très fortes chez les Burète. Elles allaient passer la Révolution et l'Empire. En 1821 encore, une des filles de Jacques Burète, Jeanne, épouse le négociant Jean Guilhou ${ }^{30}$. Or, si les Guilhou se sont installés à Bordeaux avant la Révolution, il s'agit de protestants originaires de Parnac dans le Quercy.

Le troisième courant est le plus important. Il commence dans l'Agenais avec les Sorbé, les Balguerie ou les Vignes. Pierre Sorbé devient armateur à Bordeaux vers 1740. Il se retire à la fin des années 1780 et ses trois fils exercent ensemble sous la raison Sorbé frères ou pour leur compte personnel jusqu'aux années 1830. Jean-Pierre, dit Sorbé l'aîné, est à la fois capitaine de navire et armateur, mais les trois frères pratiquent aussi la commission et possèdent en commun une raffinerie de sucre. La famille Balguerie, très prolifique, se compose de nombreuses branches. Des Balguerie étaient déjà installés à Bordeaux comme marchand avant la révocation de l'Édit de Nantes. Plusieurs générations envoient certains de leurs membres à Bordeaux. Une des branches principales, les Balguerie de Ramonds, est originaire de Clairac sur le Lot. Un de ses représentants, Jean-Pierre Balguerie, naquit 
en 1731, quelques kilomètres plus au Sud à Aiguillon, au confluent du Lot et de la Garonne. Il s'installa comme négociant à Bordeaux où il épousa la fille d'un planteur de Saint-Domingue, Marguerite Corrégeolles, qui lui donna deux fils : Jean-Isaac en 1771 et Pierre en 1778. En 1793, il abandonne le négoce et se retire sur ses terres d'Aiguillon. Pendant le Directoire, Jean-Isaac s'installe rue Leyteyre où il s'associe à Alexandre Dandiran de trois ans son aîné, sous la raison Balguerie Dandiran \& Cie. À 27 ans, le 27 ventôse an VII (18 février 1799), il épouse une cousine, Jeanne Balguerie de Raoul, qui a juste 21 ans $^{31}$. C'est la fille de Pierre Balguerie et de Catherine Baour. Le père, qui a alors 63 ans, ancien bourgeois de Bordeaux, « ci-devant seigneur de la maison noble de Raoul », s'est aussi retiré sur ses terres à Portets, sur la Garonne au Sud de Bordeaux et ne se déclare plus que "cultivateur». Le second fils Pierre, revient à Bordeaux en 1798. Marié, en 1809, à la fille du commissionnaire allemand Mathias Stuttenberg, celui qu'on nomme désormais Balguerie-Stuttenberg dirige, jusqu'à sa mort en 1825, une des sociétés les plus dynamiques de Bordeaux.

Les Vignes sont originaires du Lot-et-Garonne. Les ancêtres étaient cordiers à Saint-Sixte avant $1630^{32}$. Au XVII siècle, ils deviennent bourgeois et « marchands de rivière ». Au XVIII ${ }^{e}$ siècle, Raymond Vignes oncle, devient négociant et armateur à Bordeaux. Son frère, prénommé aussi Raymond, reste négociant à Moissac et y épouse Jacquette Gouges, la fille d'un minotier. La ville est alors, comme nous l'avons vu, un très grand centre de minoterie du Sud-Ouest. Des représentants de familles de minotiers réputés comme les Gouges, les Garisson, les Lassabathie s'installent ainsi à Bordeaux. Les Gouges et les Vignes vont se lier à plusieurs reprises et développer ainsi leurs affaires. Raymond Gouges, commissionnaire à Bordeaux à partir de 1759 épouse, en 1772, Rose, la fille de Raymond Vignes oncle. Son frère est installé au Cap-Français depuis 1766 et y dirige les expéditions de minots ${ }^{33}$. C'est dans ce milieu que Hugues Vignes naît à Moissac en 1753. Son père l'envoie auprès de son oncle avec lequel il s'associe. La société Vignes \& neveu est installée sur les allées de Tourny. À la mort de son oncle en août 1787, Vignes neveu, comme on le nomme à Bordeaux, reprend seul les affaires, dans l'hôtel des allées de Tourny qu'il avait acquis l'année précédente ${ }^{34}$. C'est alors un négociant important qui a épousé une fille du grand négoce, Anne Acquart. Comme son oncle, il accède aux honneurs : directeur à la Chambre de Commerce en 1783 et consul à la Bourse en 1785. La maison Vignes \& neveu, Hugues reste active jusqu'aux années 1820.

Le courant migratoire protestant se prolonge dans le Quercy avec les frères Guilhou. Le cadet débute comme capitaine de navire avant la Révolution, puis fait venir son frère aîné sous l'Empire pour créer la société de négoce Guilhou, Jean \& Guilhou aîné. Il continue dans l'Albigeois et le Haut-Languedoc. Les Bonnaffé et les premiers Nairac étaient venus du Tarn au début du règne de Louis XV. La famille Baour était originaire de Castres. Pierre Baour II, le premier inaugura la tradition des unions avec les Balguerie en $1746^{35}$. C'était aussi le cas des Bosc. Le père réside encore à Castres à la veille de la Révolution avec son épouse Louise Baour. En 1789, à 30 ans, Jean-Jacques, leur fils, épouse à Bordeaux la fille d'un gros marchand épicier de la place du Palais et s'installe comme négociant rue de la Rousselle. C'est surtout à partir de l'Empire qu'il devient un très grand négociant associé à son frère Alexis. Ce courant migratoire aboutit enfin dans le Bas-Languedoc avec, en particulier, Jean et Isaac Tarteyron nés à Ganges au Nord de l'Hérault, ou Jean-Étienne Balguerie junior, leur neveu, né à Montpellier. Un Guillaume Nairac, marchand épicier en gros, mais aussi négociant, né à Agde, s'installe encore à Bordeaux en 1784 et y devient vice-consul de la République de Gènes ${ }^{36}$. 

épouse ainsi en 1750, Marie Conte, la tante de Jacques Conte, de la communauté des huguenots de Saintonge. Jean Balguerie, le fils de Jacques Balguerie, né à Bordeaux en 1709, mais riche négociant de Clairac, épouse à Marseille, en 1749, Marguerite Tarteyron, née à Ganges et fille de Jean Tarteyron, marchand de Montpellier. C'est sans doute pourquoi, leur fils, Jean-Etienne Balguerie junior, qui allait devenir un des plus grands négociants de Bordeaux, y naît en 1756. En 1765, l'armateur Isaac Tarteyron se marie « au désert » avec Madeleine Boucherie et Isaac Couderc est le témoin de la future. C'est ainsi encore que, pendant le Directoire, Frédéric Gachon né à Sommières dans l'Hérault, où son père est aussi négociant, vient s'installer à Bordeaux et épouser Henriette-Manon Delorthe, issue comme Madeleine Boucherie, des milieux protestants de la vallée de la Dordogne ${ }^{37}$. C'est par l'intermédiaire des Tarteyron encore que nous découvrons des liens sur la longue durée entre Bordeaux, le Bas-Languedoc et Marseille. Une branche des Tarteyron est installée dans la cité phocéenne, y pratique le négoce jusqu'à la Révolution et maintient des relations familiales et commerciales avec la branche bordelaise ${ }^{38}$. La femme du Jean Tarteyron marchand de Montpellier au début du XviII ${ }^{e}$ siècle, se nomme Catherine Lajard. Or, durant toutes nos recherches, nous n'avons croisé qu'un seul négociant bordelais né à Marseille et il s'agit du commissionnaire de denrées coloniales et armateur Pierre Lajard. Né en 1733, il connut une belle réussite à Bordeaux et résidait sur le prestigieux cours du Chapeau Rouge au début des années 1780. En 1794, il possédait deux navires et une fortune totale estimée à la somme colossale de un million de livres. Dans son magasin, on trouvait alors des savons de Marseille et 150 balles de grosses toiles du Languedoc ${ }^{39}$. À partir de 1800, la société devint Lajard \& Rey. Or une branche des Rey faisait aussi partie du négoce marseillais et une autre était installée à Lyon. Ainsi les réseaux avec la France méridionale se maintiennent sur plusieurs générations.

\section{Hommes nouveaux ou héritiers ?}

Tous les cas que nous venons d'évoquer tendent à prouver que les héritiers dominent parmi les négociants de la France méridionale qui s'installe à Bordeaux. Mais nous venons de croiser surtout des familles connues et des hommes qui ont réussi. Seule une étude statistique sur un échantillon représentatif peut nous permettre de dresser un profil de nos migrants et de trancher véritablement. Sur les 201 contrats de méridionaux que nous livrent les archives notariales bordelaises, 163 sont de véritables négociants. Nous connaissons la profession de leurs pères pour 85 d'entre eux. Les fils de négociants représentent $45 \%$ du total. Si on y ajoute les fils de marchands et de capitaines de navires on atteint $53 \%$. La reproduction sociale est donc forte. Mais le milieu reste ouvert, beaucoup plus que pour l'ensemble des négociants bordelais où ce même total dépasse les $70 \%$. Ces négociants méridionaux venus tenter leur chance à Bordeaux sont donc davantage que leurs homologues, issus des catégories bourgeoises au sens large: professions juridiques et libérales $(14,1 \%)$ ou bourgeois sans autre précision $(8,2 \%)$. Il faut noter aussi l'importance, en leur sein, des fils de propriétaires qui atteignent $21 \% \mathrm{du}$ total contre $7 \%$ pour l'ensemble du milieu. Cela est tout à fait compréhensible pour des hommes venant, pour beaucoup, de petits bourgs encore fortement insérés dans l'économie rurale ${ }^{40}$. Nombre de pères, enfin, exercent des fonctions d'autorité : échevins, maires, juges consulaires ou présidents de tribunal de commerce. Les milieux populaires sont, en revanche, totalement absents. L'image qui se dégage est celle d'une bourgeoisie 
méridionale du commerce, des offices et de la rente envoyant un certain nombre de ses enfants dans le grand port atlantique, pour permettre à la famille de continuer son ascension en changeant d'échelle.

Les 38 contrats qui nous restent n'infirment pas cette première approche. 14 concernent des hommes qui, par leur profession, s'intègrent dans le milieu du négoce : 6 capitaines de navire, 4 agents de change, 3 courtiers et un raffineur. On compte encore 6 fils de négociants qui ne déclarent pas de profession au moment de leur mariage. Il reste 18 migrants issus d'autres milieux, mais venant à Bordeaux épouser des filles de négociants. On y trouve 9 bourgeois et propriétaires, 5 avocats, 2 officiers, 1 pharmacien et 1 garçon tailleur. C'est d'ailleurs le seul représentant de milieux modestes que nous avons croisé.

\section{Les modalités de l'installation à Bordeaux}

La modalité principale de l'installation à Bordeaux et son enracinement est une bonne alliance matrimoniale. C'est très visiblement ce que les négociants méridionaux viennent chercher. Dans de nombreux cas, il est d'ailleurs indiqué que le futur est seulement depuis quelques mois dans le port et qu'il réside chez un parent, quand ce n'est pas chez la future elle-même. Nos négociants convolent en moyenne à 33 ans, ce qui est à peine plus âgé que les autres négociants de la place (32,5 ans). Ces unions tardives conjuguées à l'éloignement familial créent une émancipation de fait. $36 \%$ des pères sont déjà morts au moment du mariage de leur fils et seuls $28,2 \%$ font le voyage pour assister à la signature du contrat. Quant aux mères, les trois quarts ne sont jamais présentes. Passée la trentaine, les hommes qui viennent à Bordeaux ont, de toute manière, intériorisé le fait que leur mariage ne doit pas menacer les intérêts de leur entreprise. Il suffit, pour s'en convaincre de s'interroger sur leurs choix matrimoniaux.

Nous connaissons bien les compagnes de nos 163 négociants méridionaux car, dans 115 cas, la profession de leur père est indiquée lors de la signature du contrat. Le constat est très clair. Presque deux fois sur trois, il s'agit de jeunes femmes issues des milieux du commerce : $56,5 \%$ du négoce et $9,5 \%$ de la marchandise. Le tiers restant est pour l'essentiel constitué de filles d'hommes de loi $(7,8 \%)$, de propriétaires $(6,1 \%)$ ou de bourgeois $(4,3 \%)$. Les plus malchanceux ou les plus modestes se rabattent sur des filles d'artisans $(2,6 \%)$. Le mariage a pour fonction d'asseoir la position du négociant qui vient de loin et de lui ouvrir l'accès à des réseaux commerciaux qu'il a peu de chances de pénétrer sans soutien familial local. On constate ainsi que dans $81 \%$ des cas, les négociants méridionaux viennent épouser à Bordeaux, une jeune femme qui y est née. La famille de la future peut, bien sûr, être originaire d'une autre région, mais elle est déjà installée dans le port depuis au moins une génération.

Les 31 mariages unissant deux méridionaux à Bordeaux qui représentent peu de chose pour les hommes pèsent d'un tout autre poids pour les femmes. C'est ainsi exactement la moitié des méridionales (31 sur 62) qui épousent à Bordeaux un négociant lui aussi originaire de ce vaste arrière-pays bordelais. Si la venue des hommes est un moyen d'intégration au négoce local, celle des femmes demeure ainsi, en partie, le moyen de maintenir des liens avec la région d'origine.

41 L'installation à Bordeaux, qui passe presque toujours par une alliance matrimoniale, peut aller jusqu'à une association avec le beau-père, association formalisée dans le contrat de 
mariage lui-même. Cela révèle alors clairement que, si l'hymen consacre l'union de deux destinées, il scelle aussi l'alliance de deux familles, de deux patrimoines et parfois de deux entreprises. Quand, durant le Directoire, Gabriel Raoux, négociant né à Nîmes, épouse Marie-Marguerite Delmas, il est ainsi précisé qu'une société sera établie entre le futur et le beau-père Antoine Delmas. Ce dernier, négociant mais aussi marchand de mouchoirs avec son beau-frère sous la raison Delmas \& Pellissier, est d'ailleurs aussi originaire de Nîmes. La future, âgée de 16 ans, est d'ailleurs née dans l'Hérault. Son mariage avec un homme de 32 ans qui devient l'associé de son père et de son oncle n'a, on le comprend, que peu à voir avec les élans du cœur ${ }^{41}$.

Pour ceux qui arrivent très jeunes, l'installation à Bordeaux commence par l'apprentissage chez un proche. Pour François Risteau, négociant originaire de SainteFoy, c'est « un parent à Bordeaux qui voulant le sortir de la misère l'appela dans sa maison. Après lui avoir fait apprendre à écrire, il en fit un commis pendant environ dix à douze ans $»^{42}$. On retrouve le même cas de figure chez les Delbos, des marchands de Domme dans le Périgord depuis le $\mathrm{XVII}^{\mathrm{e}}$ siècle $^{43}$. Le père, Jean Delbos, commerçait déjà avec Bordeaux. Ses deux fils, Jean et son frère aîné Jacques (1747-1788), se placent comme commis, avant de créer leur propre société, que l'on voit apparaitre sur l'Almanach de 1784. La société Delbos frères, installée aux Chartrons, se spécialise alors dans la vente en commission des vins du Haut-Pays. Jean-Pierre Castarède jeune, de Fleurance dans le Gers, vient, quant-à-lui, rejoindre son frère aîné, Jean, qui s'était installé quelques années auparavant. Les deux frères s'associent ensuite sous la raison Castarède frères entre 1784 et 1786. Pour Jean-Ciprien Lassabathie, né en 1728 à Moissac dans le milieu des minotiers, c'est en revanche l'inverse. Il quitte sa ville natale, car son frère aîné a hérité de l'essentiel de la fortune familiale. À l'âge de vingt ans, il tente sa chance à Bordeaux et s'y spécialise dans les productions industrielles liées à l'activité du port. C'est ainsi qu'il fonde une raffinerie de sucre, puis une verrerie sur les quais de Paludate. Dans presque tous les cas, cependant, un fort soutien de la parenté est repérable, quand il ne s'agit pas d'une véritable filière d'immigration familiale.

\section{De riches méridionaux?}

Quel genre d'hommes la France méridionale envoie-t-elle à Bordeaux? S'agit-il de modestes provinciaux venus tenter leur chance dans le grand port atlantique ou bien de représentants des meilleures familles du négoce provincial? Les apports au mariage, même s'ils ne sont qu'un reflet des niveaux de fortune, permettent de comparer ces hommes au reste des négociants bordelais. 
Figure 4 : apports cumulés au mariage dans les milieux du négoce bordelais entre 1784 et 1825 . Comparaison entre les époux issus de la France méridionale et les autres (en pourcentage et en livres tournois puis en francs).

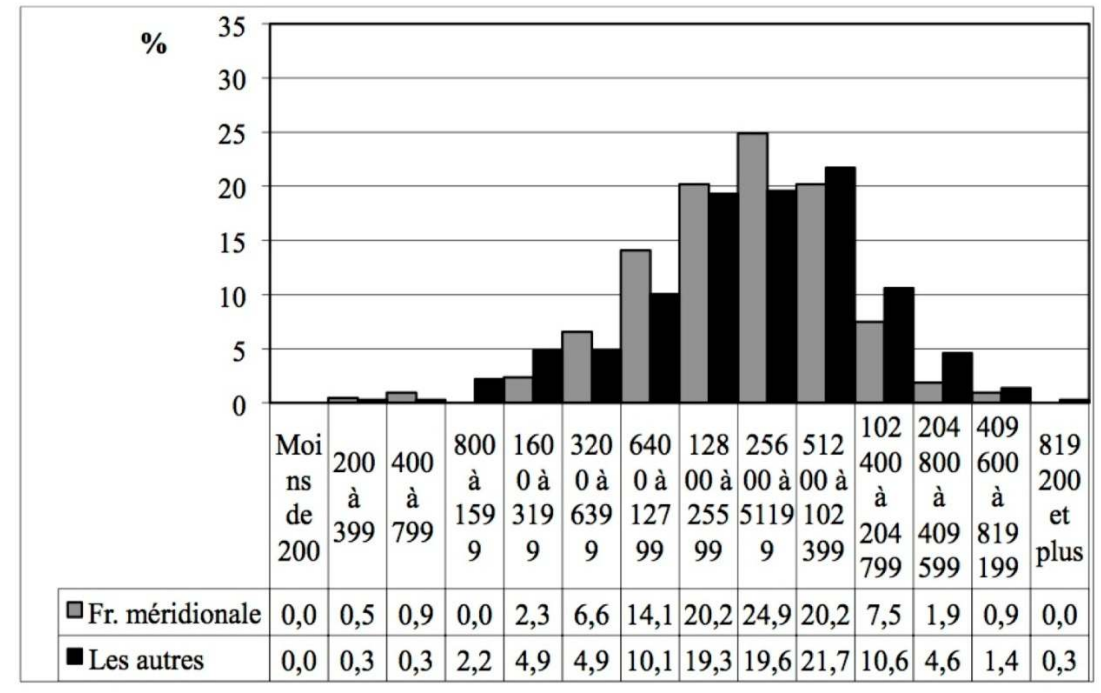

Le graphique compare les 227 contrats de méridionaux aux 428 contrats qui concernent les autres époux du négoce bordelais. Les tranches dominantes comprises entre 12.800 et $102.399 \mathrm{£t}$ ou francs sont assez comparables avec $65,3 \%$ pour les méridionaux contre $60,6 \%$ pour les autres. Au-delà, les gros contrats sont nettement moins importants pour les méridionaux avec $10,3 \%$ contre $16,9 \%$. Aucun d'ailleurs ne dépasse l'avant dernière tranche comprise entre 409.600 et $819.199 \mathrm{ft}$. Encore faut-il préciser que le plus gros atteint seulement $450.000 \mathrm{ft}$. Il s'agit du mariage entre Paul-Alexandre Nairac, né à Castres, et Pauline Bonnaffée ${ }^{44}$. Le second qui atteint $416.000 \mathrm{ft}$ concerne le négociant Joseph-Étienne Ville, né à Pamiers. Ce fils de bourgeois vient épouser à Bordeaux la fille du négociant Dominique Cabarrus ${ }^{45}$. Les Cabarrus et les Bonnaffé monopolisent les premières places. C'est, en effet, ce même Cabarrus qui, trois ans plus tôt, avait marié sa fille Jeanne à un cousin de Bayonne, le négociant Pierre-Étienne Cabarrus puisné. Ce dernier se constituait $100.000 \mathrm{ft}$ en fonds de commerce, tandis que la future apportait $70.000 \mathrm{Et}$ de $\operatorname{dot}^{46}$. C'est encore Catherine-Kelly Bonnaffé qui épouse, en 1787, l'écuyer Jean-Pierre Cornuaud de Fonbourgade né à Civrac en Bazadais. L'époux apporte 120.000 des $270.000 \mathrm{Et}$ de l'apport total ${ }^{47}$. À ces très hauts niveaux, on trouve encore Arnaud Ardenne, négociant à Pointe-à-Pitre, né à Saint-Michel de Lanes en Lauragais et qui apporte en fonds de commerce la totalité des $238000 \mathrm{ft}$ du contrat qui l'unit à la fille du négociant et marchand de farines Jean Larigaudière ${ }^{48}$. Quelques négociants méridionaux se constituent de gros fonds de commerce : $100.000 \mathrm{ft}$ ou francs pour Abraham de JosnéLéon, négociant israélite de Bayonne ${ }^{49}$, Melchior Auverny de Saint-André dans l'Hérault ${ }^{50}$, Antoine Tajan né dans le diocèse d'Auch ${ }^{51} ; 80.000$ encore pour Bernard-François Lidon de Brives où son père était aussi négociant ${ }^{52}$ ou Jules Pommez de Nay dans les BassesPyrénées $^{53}$. Dans les contrats médians, les cas les plus fréquents sont ensuite ceux de négociants méridionaux se constituant des fonds de commerce de 30.000 à $50.000 \mathrm{ft}$ puis 
francs. À l'autre extrémité, au contraire, les contrats modestes, entre 1.600 et $12.799 \mathrm{ft}$, sont un peu plus fournis que pour les autres négociants bordelais avec $23 \%$ contre 19,1\%. Enfin, les contrats pauvres, à moins de $800 \mathrm{ft}$, s'ils sont très peu nombreux, sont tout de même deux fois plus représentés que dans le reste du négoce. L'image qui se dégage est donc celle d'un milieu décalé d'une à deux tranches vers le bas, mais aussi d'un groupe relativement homogène concentré autour de la tranche de 25.600 à $51.199 \mathrm{ft}$ qui, à elle seule, représente un mariage sur quatre. Alors, des provinciaux modestes? Bien sûr que non. Les contrats exceptionnels manquent bien sûr, mais si l'on songe qu'il s'agit de négociants provenant, pour la plupart, de petites villes du Sud-Ouest, les niveaux atteints sont tout à fait remarquables. Ils prouvent que Bordeaux attire d'abord l'élite des négoces de la France méridionale ; une élite qui cherche à élargir ses horizons et à poursuivre son ascension sociale; une élite provinciale qui vient nourrir la croissance commerciale du grand emporium bordelais et entend bien en profiter.

Les mariages nous livrent les capacités à l'arrivée à Bordeaux, mais pour mesurer la réussite, il faut se pencher sur l'étude des fortunes accumulées. Si nous pouvons estimer le patrimoine de 287 négociants entre 1784 et 1825 , nous sommes loin de connaître encore tous leurs lieux de naissance, ce qui nous interdit d'établir un lien entre fortune et origines géographiques. Cependant, nous pouvons constater que nombre de ceux que nous connaissons sont loin de faire pâle figure par rapport à leurs homologues nés à Bordeaux. Fils d'un capitaine de navire et né à Biarritz, l'armateur Jacques Barthélémy Gramont de Castéra possède $315.000 \mathrm{ft}$ dans la maison Feger, Gramont \& Cie en $1784^{54}$. En 1787, Raymond Vignes de Moissac, lègue $657.000 \mathrm{ft}$, cinq maisons et sept métairies à ses cinq filles ${ }^{55}$. Jacques-Daniel Sorbé, de l'Agenais, estime sa fortune à $820.000 \mathrm{ft}$ en 1792 . Jean-Cyprien Lassabathie, de Moissac encore, estime la sienne à 430.000 en 1793. L'année suivante, la fortune de l'armateur d'origine toulousaine Louis Bertrand atteint 150 à $180.000 \mathrm{ft}^{56}$ et celle de Pierre Loriague aîné, issu d'une famille pauvre de Saint-Jean de Luz, près de $400.000^{57}$. En 1820, l'orthézien Pierre Hourquebie, né lui aussi dans une famille modeste, possède $90.000 \mathrm{~F}$ en biens immobiliers, sans compter un domaine de campagne près de Bordeaux et un château dans les Landes ${ }^{58}$. À leur mort en 1822, Jacques Burète laisse $287.000 \mathrm{~F}^{59}$ et Jean Tarteyron $364.458 \mathrm{~F}^{60}$. En 1831, enfin, les héritiers de Balguerie junior recueillent $745.000 \mathrm{~F}$.

Il est impossible, surtout dans le cadre d'un article, de présenter des dizaines de trajectoires individuelles sur une période aussi longue et à la conjoncture si heurtée. Faute d'une information suffisante et homogène sur les entreprises, nous ne pouvons dégager des comportants dominants. Nous avons choisi deux cas qui illustrent pleinement les caractéristiques générales que nous venons de dégager: rôle de l'axe garonnais et de ses principaux centres financiers, importance des filières migratoires protestantes. Le premier cas concerne un des hommes les plus illustres que le BasLanguedoc envoya à Bordeaux et le second un vaste réseau d'entreprises qui s'étendait des profondeurs de la France méridionale au Nouveau Monde.

\section{Jean-Étienne Balguerie junior : de Montpellier à l'aventure dans « les quatre parties du monde »}

47 Nous avons déjà évoqué plus haut les origines de ce Montpelliérain. Deux de ses oncles maternels l'avaient précédé à Bordeaux. Jean et Isaac Tarteyron, tous les deux nés à Ganges en 1728 et 1733. Ils établirent chacun leur propre maison d'armement dans deux 
rues du quartier de la Rousselle ${ }^{61}$. Sans pratiquer la traite, les Tarteyron commerçaient avec les Antilles et prospectaient les nouveaux marchés de l'océan Indien ${ }^{62}$. En 1765, le cadet, Jean, épousa, comme nous l'avons vu, une des filles du négociant protestant Antoine Boucherie. Par cette alliance, les Tarteyron se liaient donc aussi aux Couderc, aux Burète et aux Delorthe, c'est-à-dire au groupe protestant de la vallée de la Dordogne. En 1777, la sœur de Jean-Étienne épousa un autre négociant bordelais, Jean-Louis Baour, dont la famille, comme nous le savons, était originaire de Castres dans le Tarn. Quand Balguerie junior arriva à Bordeaux, il pouvait donc compter sur de forts soutiens.

Chose rare dans son milieu, il commença par naviguer à l'âge de 18 ans et fut reçu capitaine au long cours, à 23 ans, en 1779. Il déclarait lui-même avoir parcouru «toutes les mers des quatre parties du monde $\aleph^{63}$. De février 1783 à juin 1785, explorant les nouveaux marchés de l'océan Indien, il séjourna en Chine et fut le troisième Français à faire le tour du monde à la tête d'un navire, après Bougainville et Étienne Marchand ${ }^{64}$. C'est à cette époque que Balguerie junior acquiert une connaissance concrète des Antilles et surtout des nouveaux marchés de l'île de France, de l'Inde et de la Chine. Bien que pratiquant déjà le négoce, comme tout capitaine de navire, il n'apparaît pas sur les almanachs de l'Ancien Régime. Ce n'est qu'en 1788, qu'il s'associe au Genevois Jean-Louis Baux, spécialisé dans le commerce de "toileries $»^{65}$. Remarquons qu'il existe aussi, à la même époque, une maison Jean \& David Baux à Marseille.

En 1788, (écrit-il, quelques années plus tard), j'étais garçon, j'étais riche (...) J.L. Baux \& Cie jouissait du premier crédit, armait deux ou trois navires chaque année pour compte de divers actionnaires, avait les magasins pleins de marchandises pour compte d'autrui.

Sa fortune personnelle atteint, en effet, $263000 \mathrm{ft}$, presque entièrement investies dans trois navires pratiquant la traite et le trafic colonial avec l'île de France et le CapFrançais: L'Hippopotame, un monstre de 800 tonneaux, L'Horizon et $L^{\prime} H e c t o r^{66}$. Il avait acheté les deux premiers à Sageran \& Gautier pour lesquels il avait été capitaine. Avec cette "fortune honnête", il se situe, au $25^{\mathrm{e}}$ rang des 85 fortunes du négoce recensées entre 1784 et 1792 . C'est à ce moment qu'il s'installe vraiment en se mariant, en 1790 , avec la fille d'un négociant des Chartrons, Rion l'aîné. De 1789 à 1791, Baux \& Balguerie organise encore six armements vers l'île de France. Mais la société cesse toute activité dès la fin de 1792 et éprouve « des pertes considérables » par la suite. En novembre 1793, en pleine guerre maritime, Balguerie a encore «trois navires dans l'Inde» dont il est sans nouvelle ${ }^{67}$. Il est alors mêlé aux « intrigues mercantiles » pour sauver son associé et son oncle. Dans un mémoire de 1796, il décrit la période difficile qui commence alors: "pendant le règne de la Terreur, tout le monde sait que mon associé parut devant le tribunal de Lacombe, et mes sacrifices sont à cet égard assez notoires ». En novembre 1793, en effet, Jean-Louis Baux est arrêté par la Commission militaire. Il est acquitté et Balguerie Junior n'est pas inquiété. Comme le laisse entendre Balguerie, Lacombe a fait payer cher son indulgence : $48.000 \mathrm{ft}$ pour être exact ${ }^{68}$. La corruption du président de la Commission militaire s'ajoutait aux $113.698 \mathrm{£t}$ qu'il avait fallu verser depuis 1789 pour les différents emprunts, caisses patriotiques et aides à la municipalité concernant les subsistances et les hospices. Il servit aussi d'intermédiaire pour sauver de la guillotine son oncle Jean Tarteyron. Lacombe se contenta de $24.000 \mathrm{ft}$. En 1796, après trois années éprouvantes, sa fortune a diminué des $2 / 3$ par rapport à 1788 . Sur les $105.000 \mathrm{ft}$ de son actif brut, 60000 ont été investies dans la terre (un bien de campagne à Cissac dans le Médoc acheté en 1793) et son actif commercial est réduit à 36.550 £t. Baux se retire alors à Neuchâtel, où il décède avant 1796. 
51 Loin d'être abattu, Balguerie repart de zéro, et s'associe à un négociant parisien. Personnellement et commercialement, il se recentre sur la France méridionale. Devenu veuf, il se remarie avec Sophie Dupuy-Nozières. C'est la fille de Pierre Dupuy-Monbrun, chevalier et seigneur d'Aubignac dans le Gard. Elle va lui donner un fils et trois filles. L'alliance est sans doute un choix mûrement réfléchi dans lequel les élans du cœur n'ont pas été les seuls à compter. Il se trouve, en effet, que quelques années auparavant, Jean Tarteyron, veuf lui aussi, avait épousé la sœur de Sophie, Suzanne-Victoire DupuyNozières ${ }^{69}$. Balguerie junior se retrouvait ainsi à la fois le neveu et le beau-frère de Jean Tarteyron. C'est aussi à cette époque que son neveu, le fils d'Isaac Tarteyron, qui se nommait aussi Isaac fut élu au Conseil des Anciens, ce qui ne pouvait manquer de lui apporter quelques soutiens ${ }^{70}$. Son activité est alors celle d'un commissionnaire qui réorganise son activité autour des produits de l'arrière-pays. Dans ses magasins, il stocke un peu de vin, du tabac, des bas, des indiennes et des toiles de Toulouse et d'Agde ${ }^{71}$. Ses affaires prospèrent de nouveau pendant le Directoire et le Consulat. Il profite alors de l'apogée de la course pour redevenir armateur. Le 16 germinal an IV (4 avril 1796), il ne déclarait que quelques participations dans des navires à l'île de France. Pourtant, le 18 vendémiaire an $\mathrm{v}$ (7 octobre 1796), il vend Le Petit Expriment à l'armateur de corsaires Jacques Conte. Ce navire, dont la coque est doublée en cuivre, atteint la somme considérable de $50.000 \mathrm{Et}^{72}$. Le 12 brumaire an VII (2 novembre 1797), il lui en vend un second pour la même somme ${ }^{73}$. Il s'agit de L'Heureux, une prise anglaise. La même année, il met à la mer un navire armé de 20 pièces et mené par 218 hommes d'équipage, Le Bordelais , lequel, au cours de deux croisières de 1799 à 1801, lui rapporte plus de $750.000 \mathrm{Et}^{74}$. En 1800 , la diversification continue. Il se lance alors dans le commerce du fer avec quatre négociants, un courtier de change et un avocat. Il n'est que commanditaire, mais apporte 25.000 des $150.000 \mathrm{~F}$ du capital de cette société qui prend le nom de Jean Loubery, Pons \& $\mathrm{Cie}^{75}$. Grâce aux bénéfices fournis par la reprise des affaires, il continue à investir dans la terre. Le 24 nivôse an $\mathrm{VI}^{2}$ (13 janvier 1798), il fait l'acquisition de 16 journaux de vignes, pour $24.000 \mathrm{ft}$, au Plantier de Saint-Laurent, pratiquement dans Bordeaux ${ }^{76}$. Quand on voit ses activités, ce n'est pas un repli, mais un investissement spéculatif. Un an et demi plus tard, le 22 prairial an VII (11 juin 1799), quand il revend ce bien au négociant Jacques Ségur, pour $31.500 \mathrm{ft}$, il réalise une plus-value de $31 \%{ }^{77}$. À partir de l'annuaire de l'an VIII (fin 1799), il apparaît sous son propre nom et ce sans interruption jusqu'en 1830. Il s'est installé dans un hôtel particulier du quartier récent de la place du Champ de Mars (Jardin Public). En 1803, il monte une dernière expédition négrière de l'Angola vers Saint-Thomas aux Antilles. Mais la guerre vient de reprendre, et son navire, Le Grand-d'Alembert, est capturé. En 1808, il investit encore $30.000 \mathrm{~F}$ dans un brigantin de 179 tonneaux: La $J_{\text {Joséphine }}{ }^{78}$. Il l'engage dans la course en Martinique où il est capturé lui aussi. Cette même année, il arme deux autres corsaires La Hyène et la Revanche. Ce dernier navire ayant été capturé, il le remplace, en 1809, par L'Agile et envoie La Hyène vers l'île de France. Le blocus continental finit tout de même par réduire un temps ses activités. Le 5 mai 1810, il écrit ainsi à Daniel Lacombe : « tout est calme et mort ici, on n'espère plus aux licences anglaises, et notre gouvernement refuse aussi les siennes $»^{79}$. Il faut attendre 1813 pour qu'il lance encore deux corsaires vers l'Amérique du Nord (La Comète et Le Jason), mais il obtient tout de même quatre licences, pour expédier des vins à Londres en 1812 et $1813^{80}$.

Après 1815, il explore de nouveaux marchés pour remplacer les circuits traditionnels des Antilles, en partie périmés. Il souhaite s'insérer dans le commerce des peaux du NordOuest américain dans le but, toujours présent à son esprit, d'ouvrir la Chine au commerce 
avec l'Europe. Entre 1816 et 1819, son navire, Le Bordelais, qui lui avait déjà beaucoup rapporté dans la course, fait un tour du monde. Balguerie junior y gagne la légion d'honneur, mais ne rentre pas dans ses frais. Se procurer des fourrures de qualité sur la côte de Vancouver, les échanger en Chine contre des marchandises qui se consommaient en France et éviter ainsi l'exportation de numéraire était une idée séduisante mais difficile à rentabiliser sur un marché si lointain et déjà tenu par des compagnies anglaises et canadiennes ${ }^{81}$. Cela n'empêche pas notre négociant de devenir un grand notable de la Restauration. Sur la liste électorale de 1820, son cens atteint $3339 \mathrm{~F}$. Il est alors au $17^{\mathrm{e}}$ rang des 453 négociants électeurs. Il peut marier sa fille avec l'aristocratie du négoce. En 1823, Jeanne-Henriette épouse en effet Henri Lawton, le fils du célèbre courtier en vins Guillaume Lawton avec lequel Balguerie fait des affaires. Le futur apporte $150.000 \mathrm{~F}$ et Balguerie en donne 90.000 à sa fille. Avec $240.000 \mathrm{~F}$ d'apports cumulés, c'est un des grands mariages de la Restauration. Il se situe dans les 5,4\% des contrats du négoce qui dépassent $204.800 \mathrm{~F}$ d'apports cumulés entre 1821 et $1825^{82}$. Dans les années 1820 , Balguerie Junior reste encore actif tout en investissant dans des maisons de rapport à Bordeaux ou à Talence ${ }^{83}$, et en se lançant tardivement dans une carrière politique ${ }^{84}$. Candidat malheureux de l'opposition libérale contre les Ultras, en 1820 et 1824, il est largement élu, député de la Gironde en 1827 , avec 728 voix sur 1.107 votants ${ }^{85}$. C'est la même année, qu'il se décide à associer à ses affaires son fils Charles. Ce dernier avait épousé Jeanne-Elisabeth Burète, fille du négociant et raffineur Jacques Burète aîné que nous avons déjà croisé. Les alliances avec le groupe protestant de Bergerac se poursuivent ainsi à travers les générations. L'actif de la société monte à $450.000 \mathrm{~F}$ (300.000 F pour le père, 150.000 pour le fils). Quand il meurt, à 75 ans, le 11 mars 1831, Balguerie junior laisse une fortune considérable de $745.000 \mathrm{~F}$. Il a ainsi presque triplé son avoir en 40 ans de carrière. La traite, les trafics de l'océan Indien, la course, le commerce sous licence, Balguerie junior renouvela en permanence ses activités pour surmonter les difficultés de la période révolutionnaire et impériale, tout en maintenant des liens commerciaux et familiaux très forts avec le Bas-Languedoc.

\section{Bory frères \& Cie : un vaste réseau industriel et commercial de Montauban à Philadelphie}


Figure 5 : la maison Bory frères \&Cie, un vaste réseau industriel et commercial de Montauban à Philadelphie.

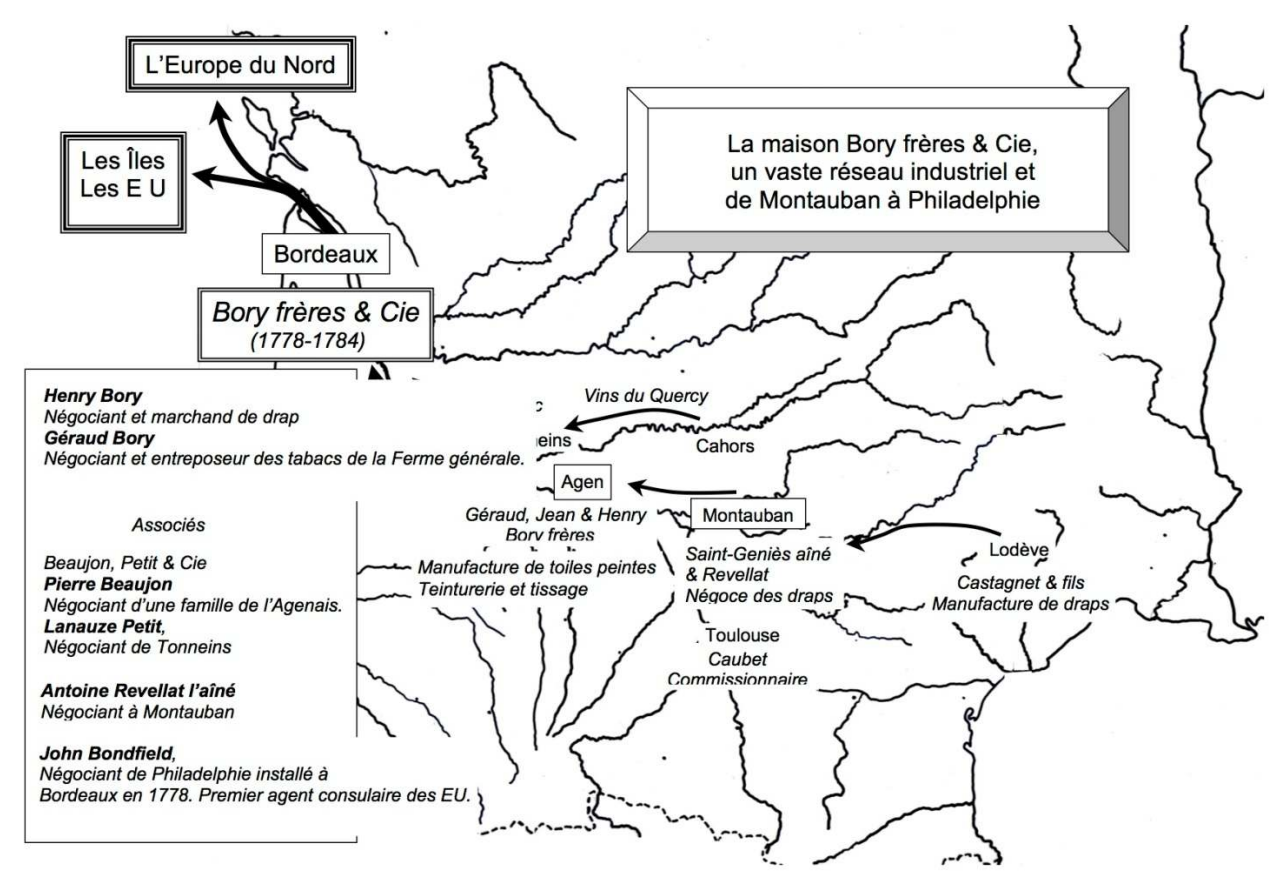

$\mathrm{Au}$ centre de ce réseau se trouve la société des frères Bory, installée rue de la Devise, au cœur des vieux quartiers du négoce. La maison Bory frères \& Cie avait été créée verbalement à Bordeaux le 3 janvier 1778. Elle associait pour un quart chacun, les frères Bory, Beaujon, Petit \& Cie, Saint-Geniès aîné \& Revellat et enfin John Bondfield. Elle nous est connue par le compte de société établi au moment de sa liquidation, en 1784, quand la société Beaujon, Petit \& Cie cesse ses activités ${ }^{86}$. À l'origine de l'entreprise on découvre la maison Géraud, Jean \& Henry Bory frères établie à Agen. Les Bory de Saint Vincent étaient une vieille famille agenaise. En 1755, ils établirent dans la ville une manufacture de draperie et une teinturerie. Très vite l'entreprise compta 300 métiers et un millier de fileuses. Elle produisait serges et étamines et utilisait des colorants provenant de Bordeaux. Elle imprimait aussi des indiennes et des mouchoirs à partir de toiles arrivant de Lorient. Le tissage vint même s'ajouter aux activités industrielles de la famille ${ }^{87}$. Henry Bory s'était installé à Bordeaux en tant que négociant mais aussi comme marchand de draperie. En 1772, son frère Géraud épouse Madeleine Journu, une des filles du grand armateur Bonaventure Journu. La famille, originaire de Lyon, était venue s'installer à Bordeaux au début du XviII ${ }^{\mathrm{e}}$ siècle. Par cette alliance, Géraud se retrouve propulsé dans le monde du haut négoce. Un de ses beaux-frères, Pierre Journu-Belille est négociant à Marseille. En 1775, son second beau-frère, Bernard Journu, épouse Geneviève-Monique Auber, fille de planteurs de Port-de-Paix à Saint-Domingue. Il se fait dès lors appeler Journu-Auber. En 1778, Géraud est encore à Agen, quand naît son fils Jean-Baptiste, le futur naturaliste et géographe ${ }^{88}$.

L'entreprise compte un associé bordelais de taille, la maison Beaujon, Petit \& Cie. Les Beaujon étaient aussi des protestants de l'Agenais qui, au XVII ${ }^{\mathrm{e}}$ siècle, vivaient de revenus liés aux offices, à la rente foncière et au commerce. La révocation de l'Édit de Nantes dispersa les enfants de Pierre Beaujon, notaire à Grateloup, jusqu'à Amsterdam. Un des fils, Jean, s'installa à Bordeaux au début du xviII ${ }^{e}$ siècle et y fit souche. Un des petits-fils 
de Jean, Nicolas, donna une ampleur sans précédent à sa maison de commerce, avant de devenir financier de la cour, directeur de la Caisse d'Escompte et d'acheter l'hôtel d'Évreux (l'Élysée) où il résida dans le faste jusqu'à sa mort. Un autre petit-fils, Pierre (1731-1786), dirigea à Bordeaux la maison Beaujon \& Petit qui nous intéresse ici et qui fut très active durant la seconde moitié du $\mathrm{XVIII}^{\mathrm{e}}$ siècle $^{89}$. Pierre Beaujon s'est associé à Lanauze Petit, un négociant de Tonneins. Beaujon \& Petit dispose ainsi d'un vaste réseau dans la vallée du Lot pour la fourniture des vins du Quercy. Elle se charge de leur revente auprès des commissionnaires de l'Europe du Nord. L'entreprise est aussi en relation avec les centres textiles du Languedoc. En 1776 et 1777, le commissionnaire toulousain Caubet lui fait parvenir des draps de la manufacture de Lodève Castagnier \& fils ${ }^{90}$. Cette solide implantation jusqu'aux confins de l'arrière-pays bordelais est renforcée grâce au troisième associé: Saint Geniès aîné \& Revellat. Représentée à Bordeaux par Antoine Revellat l'aîné, l'entreprise est en effet une maison de négoce de Montauban. Bory frères \& Cie accède ainsi au premier centre de minoterie du Sud-Ouest et s'ouvre les marchés des farines-minots pour les Antilles. L'ouverture atlantique est aussi assurée par un dernier associé: John Bondfield. Ce négociant de Philadelphie fut le premier agent consulaire des États-Unis installé à Bordeaux en 1778, avant l'arrivée, en 1790, du premier véritable consul Joseph Fenwick. Après la dissolution de la société Bory frères \& Cie, il poursuit ses affaires à Philadelphie, mais aussi à Bordeaux sous son propre nom aux Chartrons, Rue Poyenne. Sa société apparaît une dernière fois sur l'almanach de 1811. Tout laisse à penser, qu'en dehors des produits déjà évoqués, le tabac est au cœur de l'entreprise créée en 1778. Beaujon \& Petit reçevait des expéditions de tabac de la manufacture de Tonneins depuis les années $1760^{91}$. L'association avec Bondfield ne peut que faciliter l'approvisionnement en feuilles de tabac de Virginie et du Maryland, la culture étant prohibée en France depuis 1719. C'est là que l'association avec les Bory devient précieuse, car Géraud Bory tient alors l'office d'entreposeur des tabacs de la Ferme Générale à Bordeaux ${ }^{92}$. À ce titre, il est tenu d'avoir dans son magasin une ample provision des différentes espèces de tabac qui se consomment dans son district et de les revendre aux débitants à un prix fixé par arrêt du conseil ${ }^{93}$. Bory frères \& Cie contrôle ainsi cette branche d'activité, de l'approvisionnement en Amérique jusqu'à la revente au consommateur.

Dans les années 1780, c'est l'exemple même d'une entreprise qui mobilise tous les réseaux économiques, familiaux et institutionnels tissés entre Bordeaux et son arrière-pays méridional au service du grand commerce atlantique étendu jusqu'à Philadelphie.

De la fin de l'Ancien Régime à la Restauration, l'attraction bordelaise sur les milieux négociants de la France méridionale est donc tout à fait considérable. Elle génère le flux le plus important qui fournit plus de $60 \%$ des négociants non-bordelais au mariage. Il s'agit d'un flux relativement stable qui ne souffre pas des difficultés économiques de la Révolution et de l'Empire. Pour l'essentiel, c'est une immigration qui provient des ports fluviaux, des bourgs et des villes manufacturières fournissant Bordeaux en vins des Hauts-Pays, en farines-minots ou en produits manufacturés destinés au commerce avec l'Europe du Nord ou les îles.

Dans cette France méridionale, la constitution des réseaux commerciaux bordelais est en grande partie fondée sur les filières d'immigration entretenues sur plusieurs générations. Il faut souligner ici, le rôle primordial, non seulement des solidarités géographiques fondées sur les liens familiaux, mais aussi des solidarités religieuses. La communauté israélite bordelaise est ainsi renouvelée en permanence par l'apport de juifs bayonnais, 
toulousains, avignonnais et même lyonnais. Plus important encore apparaît le rôle des réformés calvinistes. De Saintonge, du Bergeracois, de l'Agenais, du Quercy, de l'Albigeois, $\mathrm{du}$ Haut et surtout du Bas-Languedoc, ils viennent en masse à Bordeaux, tout en maintenant des liens puissants avec leur pays d'origine. Les profils de ces hommes sont souvent assez comparables. Ils sont issus d'une bourgeoisie méridionale du commerce, des offices et de la rente. Ils viennent s'installer à Bordeaux chez un proche et leur enracinement passe très souvent par une alliance matrimoniale. Ils s'ouvrent ainsi les réseaux bordelais et donc les portes du grand commerce atlantique, tandis que le rôle des frères ou des parents restés au pays est essentiel pour maintenir et contrôler le commerce avec l'arrière-pays. L'étude des apports au mariage confirme cette idée qu'ils appartiennent, pour l'essentiel, à l'élite du négoce provincial pour laquelle Bordeaux constitue un tremplin permettant d'élargir les horizons et de poursuivre une ascension sociale qui avait parfois débuté deux ou trois générations plus tôt. L'étude prosopographique, qu'il faudrait pousser bien plus loin, confirme déjà que si les origines sont comparables, les destins ne sont guère généralisables. Il existe bien des manières de réussir à Bordeaux, y compris pendant et après la Révolution. Entre les simples " commissionnaires de l'intérieur " qui évitent de prendre des risques et les aventuriers, brasseurs d'affaires sans complexes, qui cherchent à ouvrir de nouvelles routes de commerce, l'écart est immense. Dans une autre perspective, il conviendrait d'approfondir le rôle de ces entreprises nébuleuses, comme celles des frères Bory, bâtissant, même pour de courtes durées, de vastes réseaux englobant de l'échelle locale au niveau international, manufacturiers, commissionnaires, armateurs et financiers.

Il est certain que sans les hommes, les entreprises et les réseaux familiaux tissés entre Bordeaux et la France méridionale, jamais le commerce du grand port atlantique n'aurait connu l'essor qui fut le sien au XVIII ${ }^{e}$ siècle. Il est tout aussi assuré que sans l'afflux permanent de jeunes acteurs du commerce provenant des élites méridionales, jamais le négoce bordelais n'aurait pu absorber le choc des guerres révolutionnaires et impériales et assurer le continuel renouvellement de ses effectifs et de ses fortunes.

\section{NOTES}

1. Jean-Pierre poussou, Bordeaux et le Sud-Ouest de la France au XVIII siècle, croissance économique et attraction urbaine, Paris, E.H.E.S.S., 1983.

2. Patrice GIBERTIE, La Famille bordelaise au milieu du XVIII ${ }^{e}$ siècle (1752-1754), TER, Bordeaux 3, 1975.

3. Philippe GARDEY, Négociants et marchands de Bordeaux de la Guerre d'Amérique à la Restauration (1780-1830), Paris, PUPS, 2009, p. 126. Prix Joseph Dutens (2009) de l'Académie des sciences morales et politiques.

4. Nous nous appuyons sur un dépouillement des contrats de mariages à partir de quatre coupes réalisées sur la totalité des notaires bordelais : 1784-1788, an III-an VIII, 1808-1812, 1821-1825.

5. Philippe GARDEY, op. cit. , p. 142.

6. Silvia MARZAGALLI, "Bordeaux au XVIII ${ }^{\mathrm{e}}$ siècle, métropole économique aquitaine?", in À la recherche de l'Aquitaine, CAHMC, Bordeaux 3, 2003, p. 136. Elle estime qu'en 1789, la part de l'«Aquitaine » dans le commerce extérieur et colonial bordelais ne dépasse pas $20 \%$ en valeur. 
7. Philippe GARDEY, «Les milieux du commerce bordelais et l'estuaire de la Gironde entre 1780 et 1830 : une relation paradoxale ", in Vivre et travailler sur les bords d'estuaire, Actes du $10^{\mathrm{e}}$ Colloque de l'Estuaire, Musée d'Aquitaine (mars 2009), Bordeaux, 2010, p. 13-41.

8. Théophile MALVESIN, Histoire du commerce de Bordeaux depuis les origines jusqu'à nos jours, t. 3, XVIII e siècle, Bordeaux, A. Bellier \& Cie Éditeurs, 1892, p. 104.

9. Jean-Pierre poussou, « Un autre regard sur les vignobles des Hauts-Pays aquitains à l'époque moderne et au XIX ${ }^{\mathrm{e}}$ siècle ", in Jean-Robert. PITTE (dir.), Le bon vin entre terroir, savoir-faire et savoirboire Actualité de la pensée de Roger Dion, CNRS éditions, 2010, p. 207.

10. Archives départementales de la Gironde [désormais Arch. dép. Gironde], 3E 21746, 11 prairial an v, étude Rauzan. À Toulouse, la maison Delmon fils \& Cie et spécialisée dans la commission des grains.

11. Arch. dép. Gironde, $3 E$ 21772, 20 février 1810, étude Rauzan père, mariage avec MarieMagdelaine de Barras, fille d'un capitaine de vaisseau, née à Saint-Domingue.

12. Archives départementales de la Haute-Garonne, acte de baptême du 28 juin 1767, paroisse de la Daurade à Toulouse.

13. Arch. dép. Gironde, $3 E$ 31370, 22 brumaire an v, étude Maillères, contrat de mariage de Clément Domecq dans lequel la société est confirmée.

14. Arch. dép. Gironde, 3L 239, $1^{\mathrm{er}}$ germinal an IV, emprunt forcé, réclamations.

15. Josette PONTET-FourniguÉ, Bayonne, un destin de ville moyenne à l'époque moderne, Biarritz, Desclée de Brouwer, 1990, p. 98.

16. Lettres de change protestées à Bordeaux sur les périodes 1784-1788; septembre 1794septembre $1800 ; 1802-1804 ; 1807-1812 ; 1821-1825$. Pour les années 1802-1804 et l'année 1807 nous avons utilisé le mémoire de L. FEILLE, Les négociants bordelais sous le Consulat et l'Empire, TER, Bordeaux 3, 1991.

17. Voir Philippe GARDEY, op. cit., p. 258-268, pour une étude complète.

18. Silvia MARZAGALLI, «Bordeaux au XVIII ${ }^{\mathrm{e}}$ siècle, métropole économique aquitaine?", op.cit.. p. 126.

19. Paul BUTEL, Les négociants bordelais, l'Europe et les Îles au XVIII siècle, Paris, Aubier-Montaigne, p. 142.

20. Paul BUTEL, op. cit., p. 101.

21. Lettre de Jean Raspay à Clossmann \& Cie, Montpellier le 16 septembre 1813. Collection personnelle de l'auteur.

22. Arch. dép. Gironde, 3E 23157, 25 fructidor an VII, étude Dufaut.

23. Arch. dép. Gironde, 3E 31425, 30 novembre 1810, étude Maillères.

24. Arch. dép. Gironde, 3E $24180,1^{\mathrm{er}}$ octobre 1822, étude Mathieu.

25. Les spécialités sont tirées de l'Almanach de commerce de Paris pour l'an XII, de DUVERNEUIL et de LA TYNNA, qui contient des listes de négociants des principales villes de France.

26. Jean-Pierre poussou, Bordeaux..., op. cit., p. 243.

27. On trouve un François Boucherie, marchand, qui se marie en 1676 avec une Jeanne Labat.

28. Arch. dép. Gironde, 3E 24890, 24 juillet 1784, étude Despiet.

29. Pierre MELLER, Les familles protestantes de Bordeaux d'après les registres de l'état civil avant 1793, Bordeaux, 1902, p. 76. Mariage avec Marie Escot du 13 juin 1784. Nombre d'informations généalogiques de cet article sont tirées de cet ouvrage.

30. Arch. dép. Gironde, 3E 24173, 20 mars 1821, étude Mathieu.

31. Arch. dép. Gironde, 3E 21749, 27 ventôse an VII, étude Rauzan.

32. Lucile BOURRACHOT, « Deux anciens villages de bateliers sur la Garonne à Saint-Sixte ", in Actes du XVIII Congrès Féd. Sav. Languedoc-Pyrénées-Gascogne et XXIV de la Féd. Hist. du Sud-Ouest, Montauban, 1972, p. 423-425.

33. Paul BUTEL, op.cit., p. 141. 
34. Arch. dép. Gironde, 3E 45608, 3 avril 1786, étude Hazera. La vente est faite pour $31.000 \mathrm{Et}$. La maison de Vignes oncle, sur ces mêmes allées de Tourny, est léguée à Rose Vignes et Raymond Gouges en 1787.

35. Paul BUTEL, Les dynasties bordelaises de Colbert à Chaban, Paris, Perrin, p. 433.

36. Arch. dép. Gironde, 3E 21721, 12 août 1784, étude Rauzan.

37. Arch. dép. Gironde, 3E 21743, 27 ventôse an IV, étude Rauzan.

38. Un Jean Tarteiron est négociant à Marseille au milieu du $\mathrm{XVIII}^{\mathrm{e}}$ siècle. Le négociant $\mathrm{L}$. $\mathrm{F}$. Tarteiron y est guillotiné en 1794 (Laurence AMÉRICI et Xavier DAUMALIN, Les dynasties marseillaises de la Révolution à nos jours, Perrin, 2010, p. 14-15). Il semble que le nom soit orthographié avec un i autour de la Méditerranée. À Bordeaux, il y a toujours un y sur les almanachs.

39. Arch. dép. Gironde, 5L 24, Commission militaire, dossier Lajard, 9 germinal an II.

40. Cependant, rien n'est jamais simple car, sous l'Ancien Régime, dans les petites cités du Midi méditerranéen, les négociants qui possèdent aussi le titre de bourgeois, privilégient cette dernière dénomination dans les actes officiels.

41. Arch. dép. Gironde, 3E 21748, 27 germinal an VI, étude Rauzan.

42. Arch. dép. Gironde, 5L 33, Commission militaire, dossier Risteau, 27 pluviôse an II.

43. Hubert Bonin, Les patrons du Second Empire, Bordeaux et la Gironde, Paris, Éd. Cénomane, 1999, p. 106-107.

44. Arch. dép. Gironde, 3E 20607, 12 avril 1787, étude Barberet.

45. Arch. dép. Gironde, 3E 17875, 15 juillet 1788, étude Hazera.

46. Arch. dép. Gironde, 3E 17867, 7 janvier 1785, étude Hazera.

47. Arch. dép. Gironde, 3E 20605, 18 février 1786. , étude Barberet.

48. Arch. dép. Gironde, 3E 17867, 7 janvier 1785, étude Hazera.

49. Arch. dép. Gironde, 3E 41378, 19 avril 1811, étude Rauzan fils. Il épouse la fille du négociant David d'Almeyda à qui son père donne $129.377 \mathrm{~F}$.

50. Arch. dép. Gironde, 3E 23194, 27 août 1822, étude Dubois. La future, fille d'un propriétaire de Port-Louis (île Maurice) se constitue $80.000 \mathrm{~F}$.

51. Arch. dép. Gironde, 3E 24718, 5 août 1788, étude Darrieux.

52. Arch. dép. Gironde, 3E 24714, 15 septembre 1786, étude Darrieux.

53. Arch. dép. Gironde, 3E 24146, 11 octobre 1812, étude Mathieu.

54. Arch. dép. Gironde, 3E 20603, 19 mars 1785, étude Barberet, liquidation d'hérédité d'ÉtienneSimon Féger-Latour. L'actif net total s'établissait à $911.192 \mathrm{ft}$ en aout 1784.

55. Arch. dép. Gironde, 3E 20608, 5 août 1787, étude Barberet, ouverture de testament.

56. Arch. dép. Gironde, 5L 6, Commission militaire, dossier Bertrand, 21 ventôse an II.

57. Arch. dép. Gironde, 5L 27, Commission militaire, dossier Loriague, 19 pluviose an II.

58. Arch. dép. Gironde, $3 Q$ 4517, enregistrement, déclarations des successions, $n^{\circ} 339$.

59. Arch. dép. Gironde, 3E 24180, inventaire, 18 octobre-9 novembre 1822, étude Mathieu et $3 Q$ 4519-20, déclarations des successions, avril 1823.

60. Arch. dép. Gironde, $3 Q$ 4519-20, enregistrement, déclarations de successions.

61. Jean Tarteyron est traduit devant la Commission militaire, mais il est acquitté en février 1794 (Arch. dép. Gironde, Commisssion militaire, 5L 35). À sa mort, à 88 ans, en 1822, il laisse une succession de $364.458 \mathrm{~F}$. Elle comprend $50.458 \mathrm{~F}$ de biens mobiliers, deux maisons et une écurie à Bordeaux $(89.000 \mathrm{~F})$, son domaine de Thouars $(80.000 \mathrm{~F})$ et un domaine à Saint-Estèphe $(145.000 \mathrm{~F})$. Il se situe au $15^{\mathrm{e}}$ rang des 99 fortunes recensées entre 1820 et 1825 (Arch. dép. Gironde, $3 Q$ 4519-20, enregistrement, déclarations de successions).

62. En 1787, Jean Tarteyron envoie Le Comte d'Estaing à l'île de France pour le compte de la compagnie des Indes de Calonne.

63. Arch. dép. Gironde, 3L 238, emprunt forcé, réclamations, 16 germinal an IV (5 avril 1796), lettre de Balguerie junior. Les citations qui suivent en sont extraites sauf indication contraire. 
64. R. CRUTCHET, "Le voyage en Chine de Balguerie Junior (1783-1785)», Revue Historique de Bordeaux et de la Gironde, 1952, p. 213-236.

65. Arch. dép. Gironde, 5L 6, Commission militaire, dossiers individuels.

66. Arch. dép. Gironde, 3L 238, emprunt forcé, réclamations, déclaration de fortune.

67. Arch. dép. Gironde, 5L 6, Commission militaire, dossiers individuels.

68. Arch. dép. Gironde, Jugement rendu par la Commission militaire spéciale qui condamne JeanBaptiste Lacombe à la peine de mort, 27 thermidor an II.

69. Le mariage est célébré en 1787 aux Salles-du-Gardon dans le Gard.

70. Adolphe ROBERT, Edgar BOURLOTON et Gaston COUGNY, Dictionnaire des parlementaires français de 1789 à 1889, t. 5, 1889, p. 371. En ligne sur http://www.assemblee-nationale.fr. Il était né en 1769 à Ganges comme son père. Dans ses tablettes manuscrites, Bernadau le juge durement : « négociant fort nul et très emporté, l'homme du parti protestant, qui n'en avait pas de meilleurs ».

71. Arch. dép. Gironde, 3L 238, emprunt forcé, réclamations, 16 germinal an IV.

72. Arch. dép. Gironde, 3E 31170, 18 vendémiaire an v, étude Maillères. Il a "oublié » de le déclarer, six mois plus tôt, ou il l'a acheté dans l'intervalle.

73. Arch. dép. Gironde, 3E 31376, 12 brumaire an VII, étude Maillères.

74. Jean de MAUPASSANT, Les Armateurs bordelais sous la Révolution et l'Empire, Balguerie Junior contre Duntzfeld, l'affaire de l'Antoinette 1799-1800, Bordeaux, Grenouilhou, 1917, p. 4. En quatre ans, Le Bordelais fit 160 prises qui auraient rapporté à ses armateurs 25 millions de francs de bénéfices !

75. Arch. dép. Gironde, 3E, 31381, 5 floréal an VIII, étude Maillères, création de société.

76. Arch. dép. Gironde, 3E 31373, 24 nivôse an vi, étude Maillères.

77. Arch. dép. Gironde, 3E 31378, 22 prairial an VII, étude Maillères.

78. Arch. dép. Gironde, 3E 31412, 17 juin 1808, étude Maillères.

79. Archives municipales de Bordeaux, Fonds Delpit 141.

80. Silvia MARZAGalli, Les Boulevards de la fraude, op. cit., annexe 1, p. 281-288 et annexe 7, p. 313-315.

81. Jean-Pierre Poussou, «Le voyage du Bordelais et le commerce des fourrures du Nord-Ouest américain : une tentative de rénovation du commerce bordelais au début de la Restauration ", in Silvia MARSAGALLI et Hubert BONIN (dir.), Négoce, ports et océans XVI-XXe siècles, Mélanges offerts à Paul Butel, P.U.B, 2000, p. 303-319.

82. Arch. dép. Gironde, 3E 24182, 22 mai 1823, étude Mathieu.

83. Arch. dép. Gironde, $3 Q$ 410, enregistrement, table des vendeurs : en 1821, une maison rue de la Merci $(9.000 \mathrm{~F})$, en 1823, une maison à Talence $(7.000 \mathrm{~F})$.

84. Il avait seulement été conseiller municipal sous l'Empire, à partir de 1806.

85. Adolphe robert, Edgar bourloton, Gaston cougny, op.cit., p. 143. Il est élu dans le $3^{\text {e }}$ arrondissement de Blaye.

86. Arch. dép. Gironde, 3E 21720, janvier 1784, étude Rauzan, compte de société au moment de la liquidation. La part de chaque associé s'élève à $53.230 \mathrm{Et}$.

87. Jean-Pierre Poussou, Bordeaux..., op. cit., p. 249.

88. Jean-Baptiste, Geneviève, Marcellin Borie de Saint-Vincent (Agen, 1778-Paris, 1846). Voir sa fiche dans le site du laboratoire de Botanique et de Mycologie de l'université de Lille 2 qui possède une partie de son herbier: http://botanique.univ-lille2.fr/lherbier-de-la-faculte/ lherbier-historique/bory-de-saint-vincent.html

89. Silvia MARZAGALLI, «De Grateloup à l'Élysée, en passant par Bordeaux : ascension sociale et mobilité de la famille Beaujon aux XVII ${ }^{\mathrm{e}}$-XVIII ${ }^{\mathrm{e}}$ siècles ", in Silvia MARZAGALLI et Hubert BONIN (dir.), op. cit., p. 19.

90. Paul BUTEL, Les négociants... op. cit., p. 99.

91. Ibid., p. 90, ainsi que "Marchands de Garonne entre la Méditerranée et l'Atlantique ", in Janine GARISSON et Marc FERRO (dir.), Une histoire de la Garonne, Ramsay, Paris, 1982, p. 344-347. La 
production était envoyée à Morlaix et Dieppe, où la Ferme la mélangeait aux tabacs de ses autres manufactures.

92. Généalogie des dirigeants de la Banque de France. http://www.genea-bdf.org/. Arbre généalogique des Journu. Le fils d'Antoine-Auguste Journu, Bernard-Auguste ayant été premier Sous-Gouverneur de la Banque de France en 1800.

93. Charles-Joseph PANCKouKE, Encyclopédie méthodique, Finances, t. 2, Paris, 1785, p. 56-57, articles " entreposeur » et "entrepôt ». L'intérêt personnel de l'entreposeur est de faire prospérer la consommation de tabac dans son arrondissement car il perçoit une remise proportionnelle au tabac écoulé. L'office peut ainsi produire jusqu'à 12.000 £t de revenus annuels.

\section{AUTEUR}

\section{PHILIPPE GARDEY}

Docteur en histoire moderne de l'Université Paris IV - Sorbonne, Centre d'Études des Mondes Moderne et Contemporain (CEMMC, EA 2958), Université Michel de Montaigne - Bordeaux III, Domaine Universitaire, 33607 PESSAC Cedex 